\title{
On algebraic properties of soft real points
}

Sabir Hussain ${ }^{1 *}$, Hurmet Fulya Akiz ${ }^{2}$ and Amlak Ibrahim Alajlan'

"Correspondence:
sabiriub@yahoo.com;
sh.hussain@qu.edu.sa
'Department of Mathematics,
College of Science, Qassim
University, Buraydah, Saudi Arabia
Full list of author information is
available at the end of the article

available at the end of the article

\begin{abstract}
In this paper, we introduce and discuss soft single points, which proceed towards soft real points by using real numbers and subsets of set of real numbers. We also define the basic operations on soft real points and explore the algebraic properties. We observe that the set of all soft real points forms a ring. Moreover, we study the soft real point metric using soft real point and explore some of its properties. We then establish a soft real point contraction fixed point theorem using soft real point metric space. It is interesting to mention that these concepts may be helpful for researchers to navigate the ideas put forth in a soft metric extension of several important fixed point theorems for metric spaces deduced from comparable existing results.
\end{abstract}

Keywords: Soft single points; Soft real points; Soft real point metric; Soft real point contraction

\section{Introduction}

Soft set theory was proposed by Molodtsov [8], for modelling vagueness and uncertainties, which is inherent in the problems of engineering, physical science, biological science, economics, social science, medical science, etc. Recently, studies on soft set theory are progressing rapidly and the topological structures of soft set and fuzzy soft set have been studied by many authors [1-12].

The idea of soft single points, which proceed towards soft real points, arose during the study of soft set theory, especially, soft topology and a soft version of the usual topology on real numbers. The existence of soft real points will enable us to define new algebraic and topological structures. Moreover, these concepts may be helpful for researchers to navigate the ideas put forth in a soft metric extension of several important fixed point theorems for metric spaces deduced from comparable existing results. It also seems that these soft real points will generalise the notions not only limited to: sets, groups, topological space etc.

\section{Preliminaries}

Let $X$ be an initial universe set, $E$ be a set of parameters, $P(X)$ be the power set of $X$ and $A \subseteq E$. First we recall some definitions.

Definition $1([8])$ A pair $(F, A)$ is called a soft set over $X$, where $F$ is a mapping given by $F: A \rightarrow P(X)$.

In other words, a soft set over $X$ is a parameterised family of subsets of the universe $X$. 
Definition 2 ([5]) Let $(F, A)$ be a soft set over $X$. If $F(x)=\emptyset$, for all $x \in A$, then $(F, A)$ is called an empty soft set, denoted by $\Phi_{A}$.

Definition 3 ([5]) Let $(F, A)$ be a soft set over $X$. If $F(x)=X$, for all $x \in A$, then $(F, A)$ is called an A-universal (absolute) soft set, denoted by $F_{\widetilde{A}}$.

Definition 4 ([7]) Let $(F, A)$ and $(G, B)$ are soft sets over $X$. Then $(F, A)$ is a soft subset of $(G, B)$, denoted by $(F, A) \widetilde{\subseteq}(G, B)$, if $F(x) \subseteq G(x)$ for all $x \in A$.

Similarly $(F, A)$ is called a soft superset of $(G, B)$ if $(G, B)$ is a soft subset of $(F, A)$. This relation is denoted by $(F, A) \supseteq(G, B)$.

Definition 5 ([7]) Two soft sets $(F, A)$ and $(G, B)$ over a common universe $X$ are called soft equal if $(F, A) \widetilde{\subseteq}(G, B)$ and $(G, B) \widetilde{\subseteq}(F, A)$.

Definition 6 ([7]) The union of two soft sets of $(F, A)$ and $(G, B)$ over the common universe $U$ is the soft set $(H, C)$, where $C=A \cup B$ and for all $x \in C$,

$$
H(x)= \begin{cases}F(x), & \text { if } x \in A-B, \\ G(x), & \text { if } x \in B-A, \\ F(x) \cup G(x), & \text { if } x \in A \cap B .\end{cases}
$$

We write $(F, A) \cup(G, B)=(H, C)$.

Definition 7 ([7]) The intersection $(H, C)$ of two soft sets $(F, A)$ and $(G, B)$ over a common universe $U$, denoted $(F, A) \cap(G, B)$, is defined as $C=A \cap B$, and $H(x)=F(x) \cap G(x)$ for all $x \in C$.

\section{Properties of soft single points}

In this section we introduce soft single points and discuss some of its properties.

Definition 8 Let $X \neq \Phi$ be a set. Then, for each $x \in X$ and $A \subseteq X$, we define a function as

$$
F_{x}^{A}:\{x\} \rightarrow P(X) \quad(x \mapsto A)
$$

Each function $F_{x}^{A}$ is called a fixed point function of the pair $x$ and $A$. The set $\left\{\left(x, F_{x}^{A}(x)\right)\right\}=$ $\{(x, A)\}$ is called a soft single point set on $X$. Also, for all $x \in X$, the set $\{(x, \Phi)\}$ is called an empty soft single point set.

The set of all soft single points is denoted by $\mathcal{S}_{X}$ and is defined as

$$
\mathcal{S}_{X}=\left\{\left\{\left(x, F_{x}^{A}(x)\right)\right\} \mid x \in X, A \subseteq X\right\} .
$$

Note that each function $F_{x}^{A}$ is unique and different for each $x \in X, A \subseteq X$, and consists of one element $(x, A)$.

Proposition 1 If $X \neq \Phi$ is a set, then the number of soft single points is determined by $\left|\mathcal{S}_{X}\right|=n 2^{n}$, where $n$ is the number of elements of $X$. 
Example 1 Let $X=\{1,2,3\}$ be a set. Then, for each $x \in X$, soft single point sets are the following:

$$
\begin{aligned}
& \{(1, X)\}, \quad\{(2, X)\}, \quad\{(3, X)\}, \quad\{(1,\{1\})\}, \quad\{(1,\{1,2\})\}, \quad\{1,\{1,3\}\}, \\
& \{(2,\{2\})\}, \quad\{(2,\{2,3\})\}, \quad\{(3,\{2,3\})\}, \quad\{(3,\{1,3\})\}, \quad\{(2,\{1,2\})\}, \\
& \{(2,\{1\})\}, \quad\{(2,\{3\})\}, \quad\{(1,\{2\})\}, \quad\{(1,\{3\})\}, \quad\{(2,\{1,3\})\}, \\
& \{(3,\{1,2\})\}, \quad\{(3,\{1\})\}, \quad\{(3,\{3\})\}, \quad\{(3,\{2\})\}, \quad\{(1,\{2,3\})\}, \\
& \{(1, \emptyset)\}, \quad\{(2, \emptyset)\}, \quad\{(3, \emptyset)\} .
\end{aligned}
$$

Definition 9 If $x=x^{\prime}$ and $A=A^{\prime}$, for $x, x^{\prime} \in X$ and $A, A^{\prime} \subseteq X$. Then we say that $\{(x, A)\}$ and $\left\{\left(x^{\prime}, A^{\prime}\right)\right\}$ are equal and this is denoted by $\{(x, A)\}=\left\{\left(x^{\prime}, A^{\prime}\right)\right\}$.

Definition 10 Consider $X$ be a set and $A \subseteq X$. Let $\mathcal{S}_{X}$ and $\mathcal{S}_{A}$ be sets of soft single point sets on $X$ and $A$, respectively. Then $\mathcal{S}_{A} \subseteq \mathcal{S}_{X}$. Moreover, $\mathcal{S}_{A}$ is called a regular subset of $\mathcal{S}_{X}$.

Definition 11 Consider $X$ to be a set and $\mathcal{S}_{X}$ to be the set of soft single point sets on $X$. Let $\mathcal{S}_{A}$ be the set of some soft single point sets on $X$. Then $\mathcal{S}_{A}$ is called an irregular subset of $\mathcal{S}_{X}$.

Proposition 2 Every regular subset is also an irregular subset.

Proposition 3 Let $X$ be a set, $\mathcal{S}_{X}$ be the set of soft single point sets and $\mathcal{S}_{A}$ be an irregular subset of $\mathcal{S}_{X}$. Then, for every $\{(a, U)\} \in \mathcal{S}_{A}$, there is an irregular subset $\mathcal{S}_{G_{a}}$ of $\mathcal{S}_{X}$ such that $\{(a, U)\} \in \mathcal{S}_{G_{a}} \subseteq \mathcal{S}_{A}$ if and only if $\mathcal{S}_{A}=\bigcup_{\{(a, U)\} \in \mathcal{S}_{A}} \mathcal{S}_{G_{a}}$.

Proof If, for every $\{(a, U)\} \in \mathcal{S}_{A}$ and $\mathcal{S}_{G_{a}}$ of $\mathcal{S}_{X}$ such that $\{(a, U)\} \in \mathcal{S}_{G_{a}} \subseteq \mathcal{S}_{A}$, then $\bigcup_{\{(a, U)\} \in \mathcal{S}_{A}} \mathcal{S}_{G_{a}} \subseteq \mathcal{S}_{A}$. Since $\{(a, U)\} \in \mathcal{S}_{G_{a}}, \mathcal{S}_{A} \subseteq \bigcup_{\{(a, U)\} \in \mathcal{S}_{A}} \mathcal{S}_{G_{a}}$, for every $\{(a, U)\} \in \mathcal{S}_{A}$. Thus $\mathcal{S}_{A}=\bigcup_{\{(a, U)\} \in \mathcal{S}_{A}} \mathcal{S}_{G_{a}}$.

On the other hand, $\{(a, U)\} \in \mathcal{S}_{A}=\bigcup_{\{(a, U)\} \in \mathcal{S}_{A}} \mathcal{S}_{G_{a}}$. This implies that there is an irregular subset $\mathcal{S}_{G_{a}}$ of $\mathcal{S}_{X}$ such that $\{(a, U)\} \in \mathcal{S}_{G_{a}} \subseteq \mathcal{S}_{A}$, for every $\{(a, U)\} \in \mathcal{S}_{A}$.

Definition 12 Let $X$ be a set and $\mathcal{S}_{X}$ be the set of soft single point sets on $X$ together with a binary operation $(*, \circ):\left(\mathcal{S}_{X} \times \mathcal{S}_{X}\right) \rightarrow \mathcal{S}_{X}$ defined as

$$
(\{(x, A)\},\{(y, B)\}) \mapsto\{(x, A)\}(*, \circ)\{(y, B)\}=\{(x * y, A \circ B)\} \in \mathcal{S}_{X}
$$

then this is called a soft single point group, if the following properties are satisfied:

(i) Closure: For any $\{(x, A)\},\{(y, B)\} \in \mathcal{S}_{X},\{(x * y, A \circ B)\}$ is also in $\mathcal{S}_{X}$.

(ii) Associativity: For any $\{(x, A)\},\{(y, B)\},\{(z, C)\} \in \mathcal{S}_{X}$, we have

$$
(\{(x, A)\}(*, \circ)\{(y, B)\})(*, \circ)\{(z, C)\}=\{(x, A)\}(*, \circ)(\{(y, B)\}(*, \circ)\{(z, C)\}) .
$$

(iii) There exists an $\{(e, I)\}$ such that

$$
\{(x, A)\}(*, \circ)\{(e, I)\}=\{(e, I)\}(*, \circ)\{(x, A)\}=\{(x, A)\}, \quad \text { for any }\{(x, A)\} .
$$

We say that $\{(e, I)\}$ is an identity element. 
(iv) Inverse: For any $\{(x, A)\}$, there exists $\{(y, B)\}$ such that

$$
\{(x, A)\}(*, \circ)\{(y, B)\}=\{(e, I)\}=\{(y, B)\}(*, \circ)\{(x, A)\} .
$$

We say that $\{(y, B)\}$ is an inverse of $\{(x, A)\}$.

We will denote soft single point group by $\left(\mathcal{S}_{X},(*, \triangle)\right)$.

Definition 13 The soft single point group is soft single point abelian, if, for any $\{(x, A)\}$, $\{(y, B)\} \in \mathcal{S}_{X},\{(x, A)\}(*, \circ)\{(y, B)\}=\{(y, B)\}(*, \circ)\{(x, A)\}$.

Definition 14 Suppose $X$ and $Y$ are set and $\mathcal{S}_{X}, \mathcal{S}_{Y}$ are sets of soft single point on $X$ and $Y$, respectively. Let $\left(\mathcal{S}_{X},(*, \triangle)\right)$ and $\left(\mathcal{S}_{y},\left(*^{\prime}, \triangle^{\prime}\right)\right)$ be soft single point groups of soft single point sets. We define a function $(f, g):\left(\mathcal{S}_{X},(*, \circ)\right) \rightarrow\left(\mathcal{S}_{y},\left(*^{\prime}, \circ^{\prime}\right)\right)$ with $\{(x, A)\} \mapsto(f, g)(\{(x, A)\})=$ $\{(f(x), g(A))\}$ thus: For any $\{(x, A)\},\left\{\left(x^{\prime}, A^{\prime}\right)\right\} \in \mathcal{S}_{X}$, we have

$$
(f, g)\left(\{(x, A)\}(*, \circ)\left\{\left(x^{\prime}, A^{\prime}\right)\right\}\right)=(f, g)(\{(x, A)\})\left(*^{\prime}, \circ^{\prime}\right)(f, g)\left(\left\{\left(x^{\prime}, A^{\prime}\right)\right\}\right) \text {. }
$$

\section{Properties of soft real points}

Definition 15 Let $\mathcal{R}$ be the set of real numbers. Then we get soft single point sets by using the fixed point functions in Definition 8. For all $r \in \mathcal{R}$ and $A \subseteq \mathcal{R}$,

$$
F_{r}^{A}:\{r\} \rightarrow P(\mathcal{R}), \quad r \mapsto A
$$

Then the set $\widetilde{\mathcal{R}}=\{\{(r, A)\} \mid r \in \mathcal{R}, A \subseteq \mathcal{R}\}$ is called the set of soft real points.

Example $2\{(1,(0,1))\},\left\{\left(\frac{1}{2},(5, \infty)\right)\right\},\{(\sqrt{3},\{0\})\}$ are the examples of soft real points.

Definition 16 If $r=r^{\prime}$ and $A=A^{\prime}$ for $r, r^{\prime} \in \mathcal{R}$ and $A, A^{\prime} \subseteq \mathcal{R}$. Then we say that soft real points $\{(r, A)\}$ and $\left\{\left(r^{\prime}, A^{\prime}\right)\right\}$ are equal and is written as $\{(r, A)\}=\left\{\left(r^{\prime}, A^{\prime}\right)\right\}$.

Now we define some operations on soft real points.

\section{Definition 17}

(i) The common sum of two soft real points $\{(r, A)\}$ and $\left\{\left(r^{\prime}, A^{\prime}\right)\right\}$ is defined by $\{(r, A)\} \widetilde{+}\left\{\left(r^{\prime}, A^{\prime}\right)\right\} \cong\left\{\left(r+r^{\prime}, A \cup A^{\prime}\right)\right\}$.

(ii) The restricted sum of two soft real points $\{(r, A)\}$ and $\left\{\left(r^{\prime}, A^{\prime}\right)\right\}$ is defined by $\{(r, A)\} \widetilde{+}_{R}\left\{\left(r^{\prime}, A^{\prime}\right)\right\} \cong\left\{\left(r+r^{\prime}, A \cap A^{\prime}\right)\right\}$.

(iii) The common difference of two soft real points $\{(r, A)\}$ and $\left\{\left(r^{\prime}, A^{\prime}\right)\right\}$ is defined by $\{(r, A)\} \simeq\left\{\left(r^{\prime}, A^{\prime}\right)\right\} \cong\left\{\left(r-r^{\prime}, A \cup A^{\prime}\right)\right\}$.

(iv) The restricted difference of two soft real points $\{(r, A)\}$ and $\left\{\left(r^{\prime}, A^{\prime}\right)\right\}$ is defined by $\{(r, A)\} \simeq_{R}\left\{\left(r^{\prime}, A^{\prime}\right)\right\} \cong\left\{\left(r-r^{\prime}, A \backslash A^{\prime}\right)\right\}$.

(v) The common product of two soft real points $\{(r, A)\}$ and $\left\{\left(r^{\prime}, A^{\prime}\right)\right\}$ is defined by $\{(r, A)\} \sim\left\{\left(r^{\prime}, A^{\prime}\right)\right\} \cong\left\{\left(r \cdot r^{\prime}, A \cup A^{\prime}\right)\right\}$.

(vi) The restricted product of two soft real points $\{(r, A)\}$ and $\left\{\left(r^{\prime}, A^{\prime}\right)\right\}$ is defined by $\{(r, A)\} \widetilde{\sim}_{R}\left\{\left(r^{\prime}, A^{\prime}\right)\right\} \cong\left\{\left(r \cdot r^{\prime}, A \cap A^{\prime}\right)\right\}$.

(vii) The common division of two soft real points $\{(r, A)\}$ and $\left\{\left(r^{\prime}, A^{\prime}\right)\right\}$ is defined by $\{(r, A)\} \stackrel{\sim}{\div}\left\{\left(r^{\prime}, A^{\prime}\right)\right\} \cong\left\{\left(r \div r^{\prime}, A \cup A^{\prime}\right)\right\}$. 
(viii) The restricted division of two soft real points $\{(r, A)\}$ and $\left\{\left(r^{\prime}, A^{\prime}\right)\right\}$ is defined by $\{(r, A)\} \stackrel{\sim}{\div}_{R}\left\{\left(r^{\prime}, A^{\prime}\right)\right\} \cong\left\{\left(r \div r^{\prime}, A \cap A^{\prime}\right)\right\}$.

(ix) The symmetric sum of two soft real points $\{(r, A)\}$ and $\left\{\left(r^{\prime}, A^{\prime}\right)\right\}$ is defined by $\left.\{(r, A)\} \widetilde{+}_{\triangle}\left\{\left(r^{\prime}, A^{\prime}\right)\right\} \stackrel{\simeq}{\cong}\left(r+r^{\prime}, A \triangle A^{\prime}\right)\right\}$, where $\triangle$ is the symmetric difference operation defined as $\left(A \triangle A^{\prime}=\left(A \backslash A^{\prime}\right) \cup\left(A^{\prime} \backslash A\right)\right)$.

(x) The symmetric difference of two soft real points $\{(r, A)\}$ and $\left\{\left(r^{\prime}, A^{\prime}\right)\right\}$ is defined by $\left.\{(r, A)\} \stackrel{\sim}{\triangle}_{\triangle}\left\{\left(r^{\prime}, A^{\prime}\right)\right\} \stackrel{\simeq}{\cong}\left(r-r^{\prime}, A \triangle A^{\prime}\right)\right\}$.

Definition 18 If $r>0$, then $\{(r, A)\}$ is called a positive soft real point and $\simeq\{(r, A)\} \cong$ $\{(-r, A)\}$ is called a negative soft real point.

Definition 19 For $\{(r, A)\},\left\{\left(r^{\prime}, A^{\prime}\right)\right\} \in \widetilde{\mathcal{R}}$, an exponential soft real point is denoted by $\{(r, A)\}^{\left\{\left(r^{\prime}, A^{\prime}\right)\right\}}$ and is defined by $\{(r, A)\}^{\left\{\left(r^{\prime}, A^{\prime}\right)\right\}} \cong\left\{\left(r^{r^{\prime}}, A \cup A^{\prime}\right)\right\}$.

Now we give some basic properties of soft real points.

Proposition 4 For $r \in \mathcal{R}$ and $A \subseteq \mathcal{R}$,

(i) $\{(r, A)\} \widetilde{+}\{(0, A)\} \cong\{(0, A)\} \widetilde{+}\{(r, A)\} \cong\{(r, A)\}$.

(ii) $\{(r, A)\} \widetilde{+}_{\mathcal{R}}\{(0, A)\} \cong\{(0, A)\} \widetilde{+}_{\mathcal{R}}\{(r, A)\} \cong\{(r, A)\}$.

(iii) $\{(r, A)\} \sim\{(1, A)\} \cong\{(1, A)\} \sim\{(r, A)\} \cong\{(r, A)\}$.

(iv) $\{(r, A)\} \widetilde{\sim}_{\mathcal{R}}\{(1, A)\} \cong\{(1, A)\} \widetilde{\sim}_{\mathcal{R}}\{(r, A)\} \cong\{(r, A)\}$.

(v) The inverse of both common sum and restricted sum of $\{(r, A)\}$ is $\simeq\{(r, A)\} \cong\{(-r, A)\}$.

(vi) The inverse of both common product and restricted product of $\{(r, A)\}$ is $\{(r, A)\}^{-1} \cong\left\{\left(r^{-1}, A\right)\right\}(r \neq 0)$.

Proof (i) From the definition of common sum,

$$
\{(r, A)\} \widetilde{\mp}\{(0, A)\} \cong\{(r+0, A \cup A)\} \cong\{(0+r, A \cup A)\} \cong\{(0, A)\} \widetilde{+}\{(r, A)\} .
$$

Also, $\{(r, A)\} \widetilde{+}\{(0, A)\} \cong\{(r+0, A \cup A)\} \cong\{(r, A)\}$.

(ii) Similar to (i).

(iii) From the definition of common product,

$$
\{(r, A)\} \sim\{(1, A)\} \cong\{(r \cdot 1, A \cup A)\} \cong\{(1 \cdot r, A \cup A)\} \cong\{(1, A)\} \sim\{(r, A)\} .
$$

Also, $\{(r, A)\} \sim\{(1, A)\} \cong\{(r \cdot 1, A \cup A)\} \cong\{(r, A)\}$.

(iv) Similar to (iii).

(v) $\{(r, A)\} \widetilde{+}\{(-r, A)\} \cong\{(-r, A)\} \widetilde{\mp}\{(r, A)\} \cong\{(0, A)\}, \quad$ and

$$
\{(r, A)\} \widetilde{+}_{\mathcal{R}}\{(-r, A)\} \cong\{(-r, A)\} \widetilde{+}_{\mathcal{R}}\{(r, A)\} \cong\{(0, A)\} .
$$

(vi) $\{(r, A)\} \sim\left\{\left(r^{-1}, A\right)\right\} \cong\left\{\left(r^{-1}, A\right)\right\} \sim\{(r, A)\} \cong\{(1, A)\}, \quad$ and

$$
\{(r, A)\} \widetilde{\sim}_{\mathcal{R}}\left\{\left(r^{-1}, A\right)\right\} \cong\left\{\left(r^{-1}, A\right)\right\} \widetilde{\sim}_{\mathcal{R}}\{(r, A)\} \cong\{(1, A)\} .
$$

The properties of exponential soft real points are as follows. 
Proposition 5 For $r, r^{\prime} \in \mathcal{R}$ and $A, A^{\prime} \subseteq \mathcal{R}$, the following are satisfied:

(i) $\left(\{(r, A)\}^{\{(s, B)\}}\right\}\left\{\left(s^{\prime}, B^{\prime}\right)\right\} \cong\{(r, A)\}\left\{(s, B)\left\{\left\{\left(s^{\prime}, B^{\prime}\right)\right\}\right.\right.$.

(ii) $\left(\{(r, A)\} \sim\left\{\left(r^{\prime}, A^{\prime}\right)\right\}\right)^{\{(s, B)\}} \cong\{(r, A)\}^{\{(s, B)\}} \sim\left\{\left(r^{\prime}, A^{\prime}\right)\right\}((s, B)\}$.

Proof For $r, r^{\prime} \in \mathcal{R}$ and $A, A^{\prime} \subseteq \mathcal{R}$,

(i) $\quad\left(\{(r, A)\}^{\{(s, B)\}}\right)^{\left\{\left(s^{\prime}, B^{\prime}\right)\right\}} \cong\left(\left\{\left(r^{s}, A \cup B\right)\right\}\right)^{\left\{\left(s^{\prime}, B^{\prime}\right)\right\}}$

$$
\begin{aligned}
& \cong\left\{\left(\left(r^{s}\right)^{s^{\prime}},(A \cup B) \cup B^{\prime}\right)\right\} \\
& \cong\left\{\left(r^{s s^{\prime}}, A \cup\left(B \cup B^{\prime}\right)\right)\right\} \\
& \cong\{(r, A)\}^{\left\{\left(s s^{\prime}, B \cup B^{\prime}\right)\right\}} \\
& \cong\{(r, A)\}^{\left\{(s, B) \Re\left\{\left(s^{\prime}, B^{\prime}\right)\right\}\right.} .
\end{aligned}
$$

(ii) $\quad\left(\{(r, A)\} \sim\left\{\left(r^{\prime}, A^{\prime}\right)\right\}\right)^{\{(s, B)\}} \cong\left(\left\{\left(r r^{\prime}, A \cup A^{\prime}\right)\right\}\right)^{\{(s, B)\}}$

$$
\begin{aligned}
& \cong\left\{\left(\left(r r^{\prime}\right)^{s},\left(A \cup A^{\prime}\right) \cup B\right)\right\} \\
& \cong\left\{\left(r^{s} r^{\prime s},(A \cup B) \cup\left(A^{\prime} \cup B\right)\right)\right\} \\
& \cong\{(r, A)\}^{\{(s, B)\}} \sim\left\{\left(r^{\prime}, A^{\prime}\right)\right\}^{\{(s, B)\}} .
\end{aligned}
$$

Definition 20 For $n \in \mathbb{N}$, the $n$th power of a soft real point $\{(r, A)\}$ is defined as

$$
(\{(r, A)\})^{n} \cong\left\{\left(r^{n}, A\right)\right\} .
$$

Proposition 6 For $n, m \in \mathbb{N}$ and $\{(r, A)\},\left\{\left(r^{\prime}, A^{\prime}\right)\right\} \in \widetilde{\mathcal{R}}$, the following properties are satisfied:

(i) $\left(\{(r, A)\}^{n}\right)^{m} \cong\{(r, A)\}^{n m}$,

(ii) $\{(r, A)\}^{n \sim}\{(r, A)\}^{m} \cong\{(r, A)\}^{n+m}$,

(iii) $\left.\left(\{(r, A)\} \widetilde{\sim}\left(r^{\prime}, A^{\prime}\right)\right\}\right)^{n} \cong\{(r, A)\}^{n} \sim\left\{\left(r^{\prime}, A^{\prime}\right)\right\}^{n}$.

Proof Consider $n, m \in \mathbb{N}$ and $\{(r, A)\},\left\{\left(r^{\prime}, A^{\prime}\right)\right\} \in \widetilde{\mathcal{R}}$.

(i) $\quad\left(\{(r, A)\}^{n}\right)^{m} \cong\left\{\left(\left(r^{n}\right)^{m}, A\right)\right\}$

$$
\begin{aligned}
& \cong\left\{\left(r^{n m}, A\right)\right\} \\
& \cong\{(r, A)\}^{n m} .
\end{aligned}
$$

(ii) $\{(r, A)\}^{n} \sim\{(r, A)\}^{m} \cong\left\{\left(r^{n}, A\right)\right\} \sim\left\{\left(r^{m}, A\right)\right\}$

$$
\begin{aligned}
& \cong\left\{\left(r^{n} r^{m}, A\right)\right\} \\
& \cong\left\{\left(r^{n+m}, A\right)\right\} \\
& \cong\{(r, A)\}^{n+m} .
\end{aligned}
$$

(iii) $\left(\{(r, A)\} \sim\left\{\left(r^{\prime}, A^{\prime}\right)\right\}\right)^{n} \cong\left\{\left(r r^{\prime}, A \cup A^{\prime}\right)\right\}^{n}$

$$
\begin{aligned}
& \cong\left\{\left(r^{n} r^{\prime n}, A \cup A^{\prime}\right)\right\} \\
& \cong\{(r, A)\}^{n} \sim\left\{\left(r^{\prime}, A^{\prime}\right)\right\}^{n} .
\end{aligned}
$$


Definition 21 Let $\{(r, A)\},\left\{\left(r^{\prime}, A^{\prime}\right)\right\} \in \widetilde{\mathcal{R}}$ with $r<r^{\prime}$ and $A \subset A^{\prime}$. Then the soft real point $\{(r, A)\}$ is a soft real point less than a soft real point $\left\{\left(r^{\prime}, A^{\prime}\right)\right\}$ or $\left\{\left(r^{\prime}, A^{\prime}\right)\right\}$ is a soft real point greater than $\{(r, A)\}$ is denoted by $\{(r, A)\} \widetilde{<}\left\{\left(r^{\prime}, A^{\prime}\right)\right\}$.

Definition 22 Let $\{(r, A)\},\left\{\left(r^{\prime}, A^{\prime}\right)\right\} \in \widetilde{\mathcal{R}}$ with $r \leq r^{\prime}$ and $A \subseteq A^{\prime}$. Then $\{(r, A)\}$ is a soft real point less than or equal to $\left\{\left(r^{\prime}, A^{\prime}\right)\right\}$ or $\left\{\left(r^{\prime}, A^{\prime}\right)\right\}$ is a soft real point greater than or equal to $\{(r, A)\}$ is denoted by $\{(r, A)\} \stackrel{\widetilde{\leq}}{\widetilde{L}}\left\{\left(r^{\prime}, A^{\prime}\right)\right\}$.

Definition 23 For $r \in \mathcal{R}$ and $A \subseteq \mathcal{R}$. The soft absolute value of the soft real point $\{(r, A)\}$ is defined by the following manner:

$$
|\{(r, A)\}| \cong\{|r|, A\} \cong \begin{cases}\{(0, A)\}, & \text { if } r=0, \\ \{(r, A)\}, & \text { if } r>0, \\ \{(-r, A)\}, & \text { if } r<0 .\end{cases}
$$

Proposition 7 Let $\{(r, A)\},\left\{\left(r^{\prime}, A^{\prime}\right)\right\}$ and $\{(s, B)\}$ be soft real points. Then the following properties are satisfied:

(i) $|\{(r, A)\}| \simeq\{(0, A)\}$.

(ii) $|\{(r, A)\}| \cong|\simeq\{(r, A)\}|$.

(iii) $\left|\{(r, A)\} \sim\left\{\left(r^{\prime}, A^{\prime}\right)\right\}\right| \cong|\{(r, A)\}| \sim\left|\left\{\left(r^{\prime}, A^{\prime}\right)\right\}\right|$.

(iv) $\left|\{(r, A)\} \stackrel{\sim}{\div}\left\{\left(r^{\prime}, A^{\prime}\right)\right\}\right| \cong|\{(r, A)\}| \stackrel{\sim}{\div}\left|\left\{\left(r^{\prime}, A^{\prime}\right)\right\}\right|$.

(v) $\{(r, A)\}^{n} \cong|\{(r, A)\}|^{n}$, for an even number $n$.

(vi) Suppose $\left|\{(r, A)\} \simeq\left\{\left(r^{\prime}, A^{\prime}\right)\right\}\right| \simeq\{(s, B)\}$, for $\{(s, B)\} \simeq\{(0, B)\}, B=A \cup A^{\prime}$. Then $\{(r, A)\} \simeq\left\{\left(r^{\prime}, A^{\prime}\right)\right\} \cong\{(s, B)\}$ or $\{(r, A)\} \simeq\left\{\left(r^{\prime}, A^{\prime}\right)\right\} \cong \simeq\{(s, B)\}$.

(vii) If $\left|\{(r, A)\} \simeq\left\{\left(r^{\prime}, A^{\prime}\right)\right\}\right| \widetilde{\leq}\{(s, B)\}$, where $B=A \cup A^{\prime}$, then $\widetilde{\simeq}\{(s, B)\} \widetilde{\simeq}\{(r, A)\} \simeq\left\{\left(r^{\prime}, A^{\prime}\right)\right\} \widetilde{\simeq}\{(s, B)\}$.

(viii) Suppose $\left|\{(r, A)\} \simeq\left\{\left(r^{\prime}, A^{\prime}\right)\right\}\right| \widetilde{\leq}\{(s, B)\}$, where $B=A \cup A^{\prime}$. Then $\{(r, A)\} \simeq\left\{\left(r^{\prime}, A^{\prime}\right)\right\} \widetilde{\simeq}\{(s, B)\}$ or $\{(r, A)\} \simeq\left\{\left(r^{\prime}, A^{\prime}\right)\right\} \widetilde{\simeq} \simeq\{(s, B)\}$.

(ix) $\left|\{(r, A)\} \widetilde{+}\left\{\left(r^{\prime}, A^{\prime}\right)\right\}\right| \widetilde{\leq}|\{(r, A)\}| \widetilde{\mp}\left|\left\{\left(r^{\prime}, A^{\prime}\right)\right\}\right|$.

(x) ||$\{(r, A)\}\left|\widetilde{\simeq}_{\Delta}\right|\left\{\left(r^{\prime}, A^{\prime}\right)\right\}|| \widetilde{\leq}\left|\{(r, A)\} \widetilde{+}_{\Delta}\left\{\left(r^{\prime}, A^{\prime}\right)\right\}\right| \widetilde{\leq}|\{(r, A)\}| \widetilde{f}_{\Delta}\left|\left\{\left(r^{\prime}, A^{\prime}\right)\right\}\right|$.

Proof (i) This is obvious.

(ii) We proof this in three cases.

- Case 1: Suppose that $\{(r, A)\} \widetilde{>}\{(0, A)\}$ then $\widetilde{\simeq}\{(r, A)\} \widetilde{<}\{(0, A)\}$. Therefore by the definition of the soft absolute value of soft real point, $|\{(r, A)\}| \cong\{(r, A)\}$, and $|\simeq\{(r, A)\}| \cong$ $\simeq(\simeq\{(r, A)\}) \cong\{(r, A)\}$. So $|\{(r, A)\}| \cong|\simeq\{(r, A)\}|$.

- Case 2: Now suppose that $\{(r, A)\} \cong\{(0, A)\}$. Therefore $\simeq\{(r, A)\} \cong\{(0, A)\}$ and clearly $|\{(r, A)\}| \simeq\{(0, A)\}$ and $|\simeq\{(r, A)\}| \cong\{(0, A)\}$. So $|\{(r, A)\}| \cong|\simeq\{(r, A)\}|$.

- Case 3: Suppose $\{(r, A)\} \widetilde{<}\{(0, A)\}$. Then $-\{(r, A)\} \widetilde{>}\{(0, A)\}$. We obtain $|\{(r, A)\}| \cong$ $\widetilde{\simeq}\{(r, A)\}$ and $|\widetilde{\simeq}\{(r, A)\}| \simeq \widetilde{\simeq}\{(r, A)\}$. So $|\{(r, A)\}| \cong|\simeq\{(r, A)\}|$.

In all three cases, we get $|\{(r, A)\}| \cong|\sim\{(r, A)\}|$.

(iii) We proof this in five cases.

- Case 1: If $r>0$ and $r^{\prime}>0$, then $\{(r, A)\} \widetilde{r}\left\{\left(r^{\prime}, A^{\prime}\right)\right\} \widetilde{>}\{(0, A)\}$.

So $\left|\{(r, A)\} \sim\left\{\left(r^{\prime}, A^{\prime}\right)\right\}\right| \cong\left\{\left(r r^{\prime}, A \cup A^{\prime}\right)\right\}$.

Furthermore, $|\{(r, A)\}| \cong\{(r, A)\}$ and $\left|\left\{\left(r^{\prime}, A^{\prime}\right)\right\}\right| \cong\left\{\left(r^{\prime}, A^{\prime}\right)\right\}$.

Therefore, $|\{(r, A)\}| \widetilde{\cdot}\left|\left\{\left(r^{\prime}, A^{\prime}\right)\right\}\right| \cong\left\{\left(r r^{\prime}, A \cup A^{\prime}\right)\right\}$. 
- Case 2: If $r<0$ and $r^{\prime}>0$, then $\{(r, A)\} \sim\left\{\left(r^{\prime}, A^{\prime}\right)\right\} \widetilde{<}\{(0, A)\}$.

Therefore, $\left|\{(r, A)\} \sim\left\{\left(r^{\prime}, A^{\prime}\right)\right\}\right| \simeq \simeq\left\{\left(r r^{\prime}, A \cup A^{\prime}\right)\right\}$.

Furthermore, $|\{(r, A)\}| \cong \simeq\{(r, A)\}$ and $\left|\left\{\left(r^{\prime}, A^{\prime}\right)\right\}\right| \cong\left\{\left(r^{\prime}, A^{\prime}\right)\right\}$.

Therefore, $|\{(r, A)\}| \widetilde{\cdot}\left|\left\{\left(r^{\prime}, A^{\prime}\right)\right\}\right| \cong\left\{\left(-r r^{\prime}, A \cup A^{\prime}\right)\right\} \cong \simeq\left\{\left(r r^{\prime}, A \cup A^{\prime}\right)\right\}$.

Similarly, for $r>0$ and $r^{\prime}<0$.

- Case 3: If $r<0$ and $r^{\prime}<0$, then $\{(r, A)\} \sim\left\{\left(r^{\prime}, A^{\prime}\right)\right\} \sim 0$. Thus, $\left|\{(r, A)\} \sim\left\{\left(r^{\prime}, A^{\prime}\right)\right\}\right| \cong$ $\left\{\left(r r^{\prime}, A \cup A^{\prime}\right)\right\}$. Furthermore, $|\{(r, A)\}| \cong \simeq\{(r, A)\}$ and $\left|\left\{\left(r^{\prime}, A^{\prime}\right)\right\}\right| \cong \simeq\left\{\left(r^{\prime}, A^{\prime}\right)\right\}$.

Therefore, $|\{(r, A)\}| \sim\left|\left\{\left(r^{\prime}, A^{\prime}\right)\right\}\right| \cong(\widetilde{\simeq}\{(r, A)\}) \sim\left(\widetilde{\sim}\left\{\left(r^{\prime}, A^{\prime}\right)\right\}\right) \cong\left\{\left(r r^{\prime}, A \cup A^{\prime}\right)\right\}$.

- Case 4: If $r=0$ and $r^{\prime}=0$, then $\{(r, A)\} \sim\left\{\left(r^{\prime}, A^{\prime}\right)\right\} \cong\left\{\left(0, A \cup A^{\prime}\right)\right\}$.

So $\left|\{(r, A)\} \sim\left\{\left(r^{\prime}, A^{\prime}\right)\right\}\right| \cong\left\{\left(0, A \cup A^{\prime}\right)\right\}$. Further $|\{(r, A)\}| \cong\{(0, A)\}$ and $\left|\left\{\left(r^{\prime}, A^{\prime}\right)\right\}\right| \cong\left\{\left(0, A^{\prime}\right)\right\}$.

Therefore, $|\{(r, A)\}| \sim\left|\left\{\left(r^{\prime}, A^{\prime}\right)\right\}\right| \cong\left\{\left(0, A \cup A^{\prime}\right)\right\}$.

- Case 5: If $r=0$ and $r^{\prime}>0$, then $\{(r, A)\} \sim\left\{\left(r^{\prime}, A^{\prime}\right)\right\} \cong\{(0, A)\} \sim\left\{\left(r^{\prime}, A^{\prime}\right)\right\} \cong\left\{\left(0, A \cup A^{\prime}\right)\right\}$.

Therefore, $\left|\{(r, A)\} \sim\left\{\left(r^{\prime}, A^{\prime}\right)\right\}\right| \cong\left|\left\{\left(0, A \cup A^{\prime}\right)\right\}\right| \cong\left\{\left(0, A \cup A^{\prime}\right)\right\}$.

Further, $|\{(r, A)\}| \cong\{(0, A)\}$ and $\left|\left\{\left(r^{\prime}, A^{\prime}\right)\right\}\right| \cong\left\{\left(r^{\prime}, A^{\prime}\right)\right\}$. Thus $|\{(r, A)\}| \sim\left|\left\{\left(r^{\prime}, A^{\prime}\right)\right\}\right| \cong$ $\{(0, A)\} \sim\left\{\left(r^{\prime}, A^{\prime}\right)\right\} \cong\left\{\left(0, A \cup A^{\prime}\right)\right\}$.

Similarly if $\left(r=0\right.$ and $\left.r^{\prime}<0\right)$ or $\left(r>0\right.$ and $\left.r^{\prime}=0\right)$ or $\left(r<0\right.$ and $\left.r^{\prime}=0\right)$.

In all these cases, we get $\left|\{(r, A)\} \sim\left\{\left(r^{\prime}, A^{\prime}\right)\right\}\right| \cong|\{(r, A)\}| \sim\left|\left\{\left(r^{\prime}, A^{\prime}\right)\right\}\right|$.

(iv) Similarly to (iii).

(v) We know that $\{(r, A)\}^{n}=\left\{\left(r^{n}, A\right)\right\}>0$, if $n$ is even. Then

$$
\begin{aligned}
\{(r, A)\}^{n} & \cong\left|\{(r, A)\}^{n}\right| \cong \mid \underbrace{|\{(r, A)\} \sim\{(r, A)\} \sim \ldots \sim\{(r, A)\}|}_{n \text { times }} \\
& \cong \underbrace{|\{(r, A)\}| \sim|\{(r, A)\}| \sim \ldots \sim|\{(r, A)\}|}_{n \text { times }}=|\{(r, A)\}|^{n} .
\end{aligned}
$$

(vi) If

$$
\begin{aligned}
\left|\{(r, A)\} \simeq\left\{\left(r^{\prime}, A^{\prime}\right)\right\}\right| \cong\{(s, B)\} & \Leftrightarrow\left|\left\{\left(r-r^{\prime}, A \cup A^{\prime}=B\right)\right\}\right| \cong\{(s, B)\} \\
& \Leftrightarrow \quad\left\{\left(\left|r-r^{\prime}\right|\right), B\right\} \cong\{(s, B)\} \Leftrightarrow\left|r-r^{\prime}\right|=s .
\end{aligned}
$$

Then $r-r^{\prime}=s$ or $-\left(r-r^{\prime}\right)=s \Leftrightarrow\left(r-r^{\prime}\right)=-s$.

So $\{(r, A)\} \simeq\left\{\left(r^{\prime}, A^{\prime}\right)\right\} \cong\{(s, B)\}$ or $\{(r, A)\} \simeq\left\{\left(r^{\prime}, A^{\prime}\right)\right\} \cong \simeq\{(s, B)\}$.

(vii) If

$$
\begin{aligned}
\left|\{(r, A)\} \simeq\left\{\left(r^{\prime}, A^{\prime}\right)\right\}\right| \widetilde{\leq}\{(s, B)\} & \Leftrightarrow\left|\left\{\left(r-r^{\prime}, A \cup A^{\prime}=B\right)\right\}\right| \widetilde{\leq}\{(s, B)\} \\
& \Leftrightarrow\left\{\left(\left|r-r^{\prime}\right|\right), B\right\} \widetilde{\leq}\{(s, B)\} \quad \Leftrightarrow \quad\left|r-r^{\prime}\right| \leq s,
\end{aligned}
$$

then we get $-s \leq r-r^{\prime} \leq s$.

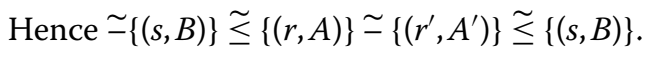

(viii) Consider

$$
\begin{aligned}
\left|\{(r, A)\} \simeq\left\{\left(r^{\prime}, A^{\prime}\right)\right\}\right| \simeq\{(s, B)\} & \Leftrightarrow\left|\left\{\left(r-r^{\prime}, A \cup A^{\prime}=B\right)\right\}\right| \widetilde{\geq}\{(s, B)\} \\
& \Leftrightarrow \quad\left\{\left|r-r^{\prime}\right|, B\right\} \simeq\{(s, B)\} \quad \Leftrightarrow \quad\left|r-r^{\prime}\right| \geq s .
\end{aligned}
$$


Thus we get $r-r^{\prime} \geq s$ or $r-r^{\prime} \leq-s$. Hence $\{(r, A)\} \simeq\left\{\left(r^{\prime}, A^{\prime}\right)\right\} \simeq\{(s, B)\}$ or $\{(r, A)\} \simeq$ $\left\{\left(r^{\prime}, A^{\prime}\right)\right\} \stackrel{\simeq}{\simeq}\{(s, B)\}$.

(ix) For any two soft real points, we have

$$
\begin{aligned}
& \simeq|\{(r, A)\}| \widetilde{\leq}\{(r, A)\} \widetilde{\leq}|\{(r, A)\}|, \\
& \simeq\left|\left\{\left(r^{\prime}, A^{\prime}\right)\right\}\right| \widetilde{\leq}\left\{\left(r^{\prime}, A^{\prime}\right)\right\} \widetilde{\leq}\left|\left\{\left(r^{\prime}, A^{\prime}\right)\right\}\right| .
\end{aligned}
$$

Therefore,

$$
\widetilde{\simeq}\left(|\{(r, A)\}| \widetilde{+}\left|\left\{\left(r^{\prime}, A^{\prime}\right)\right\}\right|\right) \widetilde{\leq}\{(r, A)\} \widetilde{+}\left\{\left(r^{\prime}, A^{\prime}\right)\right\} \widetilde{\leq}\left(|\{(r, A)\}| \widetilde{+}\left|\left\{\left(r^{\prime}, A^{\prime}\right)\right\}\right|\right) .
$$

Hence $\left|\{(r, A)\} \widetilde{+}\left\{\left(r^{\prime}, A^{\prime}\right)\right\}\right| \widetilde{\leq}|\{(r, A)\}| \widetilde{+}\left|\left\{\left(r^{\prime}, A^{\prime}\right)\right\}\right|$.

(x) First we prove right hand side. For any two soft real points we have

$$
\begin{aligned}
& \widetilde{\simeq}|\{(r, A)\}| \widetilde{\leq}\{(r, A)\} \stackrel{\widetilde{\leq}}{\simeq}|\{(r, A)\}|, \\
& \widetilde{\simeq}\left|\left\{\left(r^{\prime}, A^{\prime}\right)\right\}\right| \widetilde{\leq}\left\{\left(r^{\prime}, A^{\prime}\right)\right\} \widetilde{\leq}\left|\left\{\left(r^{\prime}, A^{\prime}\right)\right\}\right| .
\end{aligned}
$$

Then

$$
\begin{aligned}
\widetilde{\simeq}\left(|\{(r, A)\}| \widetilde{+}_{\Delta}\left|\left\{\left(r^{\prime}, A^{\prime}\right)\right\}\right|\right) & \widetilde{\leq}\{(r, A)\} \widetilde{+}_{\Delta}\left\{\left(r^{\prime}, A^{\prime}\right)\right\} \\
& \widetilde{\leq}\left(|\{(r, A)\}| \widetilde{+}_{\Delta}\left|\left\{\left(r^{\prime}, A^{\prime}\right)\right\}\right|\right) .
\end{aligned}
$$

Therefore,

$$
\left|\{(r, A)\} \widetilde{+}_{\Delta}\left\{\left(r^{\prime}, A^{\prime}\right)\right\}\right| \widetilde{\leq}|\{(r, A)\}| \widetilde{+}_{\Delta}\left|\left\{\left(r^{\prime}, A^{\prime}\right)\right\}\right| .
$$

Now we prove the left hand side.

$$
|\{(r, A)\}| \cong\left|\{(r, A)\} \widetilde{+}_{\Delta}\left\{\left(r^{\prime}, A^{\prime}\right)\right\} \widetilde{\simeq}_{\Delta}\left\{\left(r^{\prime}, A^{\prime}\right)\right\}\right| .
$$

This implies that

$$
\begin{aligned}
|\{(r, A)\}| & \widetilde{\leq}\left|\{(r, A)\} \widetilde{\mp}_{\Delta}\left\{\left(r^{\prime}, A^{\prime}\right)\right\}\right| \widetilde{\mp}_{\Delta}\left|\widetilde{\tau}_{\Delta}\left\{\left(r^{\prime}, A^{\prime}\right)\right\}\right| \\
& \cong\left|\{(r, A)\} \widetilde{\mp}_{\Delta}\left\{\left(r^{\prime}, A^{\prime}\right)\right\}\right| \widetilde{\mp}_{\Delta}\left|\left\{\left(r^{\prime}, A^{\prime}\right)\right\}\right| .
\end{aligned}
$$

This implies that

$$
\begin{aligned}
& |\{(r, A)\}| \widetilde{\sim}_{\Delta}\left|\left\{\left(r^{\prime}, A^{\prime}\right)\right\}\right| \widetilde{\leq}\left|\{(r, A)\} \widetilde{\mp}_{\Delta}\left\{\left(r^{\prime}, A^{\prime}\right)\right\}\right| \widetilde{\mp}_{\Delta}\left|\left\{\left(r^{\prime}, A^{\prime}\right)\right\}\right| \widetilde{\sim}_{\Delta}\left|\left\{\left(r^{\prime}, A^{\prime}\right)\right\}\right| \\
& \quad \Longrightarrow \quad|\{(r, A)\}| \widetilde{\sim}_{\Delta}\left|\left\{\left(r^{\prime}, A^{\prime}\right)\right\}\right| \widetilde{\leq}\left|\{(r, A)\} \widetilde{\mp}_{\Delta}\left\{\left(r^{\prime}, A^{\prime}\right)\right\}\right| .
\end{aligned}
$$

Now

$$
\begin{aligned}
\left|\left\{\left(r^{\prime}, A^{\prime}\right)\right\}\right| & \cong\left|\{(r, A)\} \widetilde{\sim}_{\Delta}\{(r, A)\} \widetilde{\mp}_{\Delta}\left\{\left(r^{\prime}, A^{\prime}\right)\right\}\right| \\
& \simeq\left|\{(r, A)\} \widetilde{\mp}_{\Delta}\left\{\left(r^{\prime}, A^{\prime}\right)\right\}\right| \widetilde{\mp}_{\Delta}\left|\widetilde{\mp}_{\Delta}\{(r, A)\}\right| \\
& \cong\left|\{(r, A)\} \widetilde{\mp}_{\Delta}\left\{\left(r^{\prime}, A^{\prime}\right)\right\}\right| \widetilde{\mp}_{\Delta}|\{(r, A)\}|
\end{aligned}
$$




$$
\begin{aligned}
& \Rightarrow \quad\left|\left\{\left(r^{\prime}, A^{\prime}\right)\right\}\right| \widetilde{\simeq}_{\Delta}|\{(r, A)\}| \widetilde{\leq}\left|\{(r, A)\} \widetilde{+}_{\Delta}\left\{\left(r^{\prime}, A^{\prime}\right)\right\}\right| \\
& \Longrightarrow \quad|\{(r, A)\}| \widetilde{\simeq}_{\Delta}\left|\left\{\left(r^{\prime}, A^{\prime}\right)\right\}\right| \widetilde{\simeq} \widetilde{\simeq}_{\Delta}\left|\{(r, A)\} \widetilde{f}_{\Delta}\left\{\left(r^{\prime}, A^{\prime}\right)\right\}\right| .
\end{aligned}
$$

From (1) and (2), we get

$$
||\{(r, A)\}\left|\widetilde{\sim}_{\Delta}\right|\left\{\left(r^{\prime}, A^{\prime}\right)\right\}|| \widetilde{\leq}\left|\{(r, A)\} \widetilde{\mp}_{\Delta}\left\{\left(r^{\prime}, A^{\prime}\right)\right\}\right| .
$$

Definition 24 Let $\{(r, A)\}$ be a soft real point. Then the $n$th $\operatorname{root} \sqrt[n]{\{(r, A)\}}$ of $\{(r, A)\}$ is defined as

$$
\sqrt[n]{\{(r, A)\}} \cong\{(\sqrt[n]{r}, A)\}, \quad \text { where } n \in \mathbb{N} .
$$

Proposition 8 Let $\{(r, A)\},\left\{\left(r^{\prime}, A^{\prime}\right)\right\}$ be soft real points and $n, m$ be natural numbers. Then the following are satisfied:

(i) $\sqrt[n]{\{(r, A)\}^{m}} \cong\{(r, A)\}^{\frac{m}{n}}$.

(ii) $\sqrt[n]{\{(r, A)\}^{n}} \cong|\{(r, A)\}|$, where $n$ is even.

(iii) $\sqrt[n]{\{(r, A)\}^{n}} \cong\{(r, A)\}$, where $n$ is odd.

(iv) $\sqrt[m]{\sqrt[n]{\left\{\left(r^{\prime}, A^{\prime}\right)\right\}}} \cong \sqrt[m n]{\left\{\left(r^{\prime}, A^{\prime}\right)\right\}}$.

Proof

(i) $\sqrt[n]{\{(r, A)\}^{m}} \cong \sqrt[n]{\left\{\left(r^{m}, A\right)\right\}} \cong\left\{\left(r^{m}, A\right)\right\}^{\frac{1}{n}}$

$$
\cong\left\{\left(\left(r^{m}\right)^{\frac{1}{n}}, A\right)\right\} \cong\left\{\left(r^{\frac{m}{n}}, A\right)\right\} \cong\{(r, A)\}^{\frac{m}{n}} .
$$

(ii) If $n$ is even, we have $\{(r, A)\}^{n}=\{(-r, A)\}^{n}=(\widetilde{\simeq}\{(r, A)\})^{n}$. This implies that soft real points $\{(r, A)\}^{n}$ and $(\widetilde{\simeq}\{(r, A)\})^{n}$ are the $n$th roots of $\{(r, A)\}^{n}$. Now

$$
\begin{aligned}
& \sqrt[n]{\{(r, A)\}^{n}} \cong\{(r, A)\}, \quad \text { if } r>0, \\
& \sqrt[n]{\{(r, A)\}^{n}} \cong\{(0, A)\}, \quad \text { if } r=0, \\
& \sqrt[n]{\{(-r, A)\}^{n}} \cong\{(r, A)\}, \quad \text { if } r<0 .
\end{aligned}
$$

Hence $\sqrt[n]{\{(r, A)\}^{n}} \cong|\{(r, A)\}|$.

(iii) Now $\{(r, A)\}^{n} \widetilde{\neq}\{(-r, A)\}^{n}$, where $n$ is odd. Then $\sqrt[n]{\{(r, A)\}^{n}} \cong\{(r, A)\}^{n}$, if $r>0$ and $\sqrt[n]{\{(-r, A)\}^{n}} \cong\{(-r, A)\}^{n}$, if $r<0$. Thus $\sqrt[n]{\{(r, A)\}^{n}} \cong\{(r, A)\}$, where $n$ is odd.

$$
\text { (iv) } \begin{aligned}
\sqrt[m]{\sqrt[n]{\left\{\left(r^{\prime}, A^{\prime}\right)\right\}}} \cong & \sqrt[m]{\left\{\left(\sqrt[n]{r^{\prime}}, A^{\prime}\right)\right\}} \cong \sqrt[m]{\left\{\left(r^{\prime} \frac{1}{n}, A^{\prime}\right)\right\}} \cong\left\{\left(\left(r^{\prime} \frac{1}{n}\right)^{\frac{1}{m}}, A^{\prime}\right)\right\} \\
& \cong\left\{\left(r^{\prime} \frac{1}{n m}, A^{\prime}\right)\right\} \cong\left\{\left(\sqrt[m n]{r^{\prime}}, A^{\prime}\right)\right\} \cong \sqrt[m n]{\left\{\left(r^{\prime}, A^{\prime}\right)\right\}} .
\end{aligned}
$$

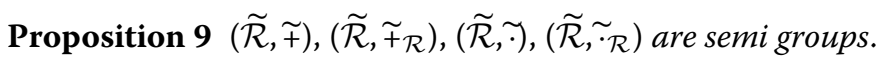

Proof To prove $(\widetilde{\mathcal{R}}, \widetilde{\mp})$ is a semi group, we show that $\widetilde{\mathcal{R}}$ is closed under $\widetilde{+}$ and satisfies the associative property. For $\{(r, A)\},\{(s, B)\},\{(t, C)\} \in \widetilde{\mathcal{R}}$,

(i) $\{(r, A)\} \widetilde{+}\{(s, B)\} \cong\{(r+s, A \cup B)\} \in \widetilde{\mathcal{R}} \quad(\widetilde{\mathcal{R}}$ is closed under $\widetilde{+}$. 
(ii) $\quad(\{(r, A)\} \widetilde{+}\{(s, B)\}) \widetilde{+}\{(t, C)\}$

$$
\begin{aligned}
& \cong\{(r+s, A \cup B)\} \widetilde{+}\{(t, C)\} \\
& \cong\{(r+s+t,(A \cup B) \cup C)\} \cong\{(r+(s+t), A \cup(B \cup C))\} \\
& \cong\{(r, A)\} \widetilde{+}\{(s+t, B \cup C)\} \cong\{(r, A)\} \widetilde{+}(\{(s, B)\} \widetilde{+}\{(t, C)\}) .
\end{aligned}
$$

So $(\widetilde{\mathcal{R}}, \widetilde{+})$ is semi group. Similarly for $\left(\widetilde{\mathcal{R}}, \widetilde{\mp}_{\mathcal{R}}\right)$.

To prove $(\widetilde{\mathcal{R}}, \widetilde{\sim})$ is semi group, we show that $\widetilde{\mathcal{R}}$ is closed under $\widetilde{r}$ and satisfies the associative property.

Let $\{(r, A)\},\{(s, B)\},\{(t, C)\} \in \widetilde{\mathcal{R}}$, then

(i) $\{(r, A)\} \sim\{(s, B)\} \cong\{(r \cdot s, A \cup B)\} \in \widetilde{\mathcal{R}}$,

for every $\{(r, A)\},\{(s, B)\} \in \widetilde{\mathcal{R}}(\widetilde{\mathcal{R}}$ is closed under $\widetilde{*})$.

(ii) $\quad(\{(r, A)\} \sim\{(s, B)\}) \sim\{(t, C)\}$

$$
\begin{aligned}
& \cong\{(r \cdot s, A \cup B)\} \sim\{(t, C)\} \\
& \cong\{(r \cdot s \cdot t,(A \cup B) \cup C)\} \cong\{(r \cdot(s \cdot t), A \cup(B \cup C))\} \\
& \cong\{(r, A)\} \sim\{(s \cdot t, B \cup C)\} \cong\{(r, A)\} \sim(\{(s, B)\} \sim\{(t, C)\}) .
\end{aligned}
$$

Therefore, $(\widetilde{\mathcal{R}}, \widetilde{\sim})$ is semi group. Similarly for $\left(\widetilde{\mathcal{R}}, \widetilde{\bullet}_{\mathcal{R}}\right)$.

Proposition $10\left(\widetilde{\mathcal{R}}, \widetilde{+}_{\Delta}\right)$ is an abelian group.

Proof For all $\{(r, A)\},\{(s, B)\},\{(t, C)\} \in \widetilde{\mathcal{R}}$,

(i) $\{(r, A)\} \widetilde{+}_{\Delta}\{(s, B)\} \cong\{(r+s, A \triangle B)\} \in \widetilde{\mathcal{R}}$.

So, $\widetilde{\mathcal{R}}$ is closed under $\widetilde{+}_{\Delta}$.

(ii) $\quad\left(\{(r, A)\} \widetilde{+}_{\Delta}\{(s, B)\}\right) \widetilde{\mp}_{\Delta}\{(t, C)\}$

$$
\begin{aligned}
& \cong\{(r+s, A \Delta B)\} \widetilde{+}_{\Delta}\{(t, C)\} \\
& \cong\{(r+s+t,(A \Delta B) \Delta C)\} \cong\{(r+(s+t), A \Delta(B \Delta C))\} \\
& \cong\{(r, A)\} \widetilde{+}_{\Delta}\{(s+t, B \triangle C)\} \cong\{(r, A)\} \widetilde{\mp}_{\Delta}\left(\{(s, B)\} \widetilde{+}_{\Delta}\{(t, C)\}\right) .
\end{aligned}
$$

So, $\widetilde{\mathcal{R}}$ is associative under $\widetilde{+}_{\Delta}$.

(iii) The identity soft real point in $\widetilde{\mathcal{R}}$ under $\widetilde{+}_{\Delta}$ is $\{(0, \Phi)\}$. That is,

$$
\begin{aligned}
\{(r, A)\} \widetilde{\mp}_{\triangle}\{(0, \Phi)\} \cong & \{(r+0, A \triangle \Phi)\} \cong\{(r, A)\} \\
& =\{(0, \Phi)\} \widetilde{\mp}_{\triangle}\{(r, A)\}, \quad \text { for every }\{(r, A)\} \in \widetilde{\mathcal{R}} .
\end{aligned}
$$

(iv) The inverse of soft real point $\{(r, A)\}$ under $\widetilde{\mp}_{\Delta}$, is $\widetilde{\simeq}\{(r, A)\} \cong\{(-r, A)\}$. That is,

$$
\begin{aligned}
\{(r, A)\} \widetilde{+}_{\triangle}\{(-r, A)\} & \cong\{(r-r, A \triangle A)\} \cong\{(0, \Phi)\} \\
& \cong\{(-r, A)\} \widetilde{+}_{\triangle}\{(r, A)\}, \quad \text { for every }\{(r, A)\} \in \widetilde{\mathcal{R}} .
\end{aligned}
$$


(v) $\{(r, A)\} \widetilde{+}_{\Delta}\{(s, B)\} \cong\{(r+s, A \triangle B)\} \cong\{(s+r, B \triangle A)\} \cong\{(s, B)\} \widetilde{f}_{\triangle}\{(r, A)\}$.

So, $\widetilde{\mathcal{R}}$ is abelian under $\widetilde{+}_{\Delta}$.

Thus $\left(\widetilde{\mathcal{R}}, \widetilde{+}_{\triangle}\right)$ is an abelian group.

Proposition $11(\widetilde{\mathcal{R}}, \widetilde{+}),\left(\widetilde{\mathcal{R}}, \widetilde{\mp}_{\mathcal{R}}\right),(\widetilde{\mathcal{R}}, \widetilde{\sim}),\left(\widetilde{\mathcal{R}}, \sim_{\mathcal{R}}\right)$ are not groups.

Proof $(\widetilde{\mathcal{R}}, \widetilde{+})$ is not a group, because, for any soft real point $\{(r, A)\} \in \widetilde{\mathcal{R}}$, we have two inverses $\simeq\{(r, A)\}$ and $\simeq\{(r, \Phi)\}$. We have

$$
\begin{aligned}
& \{(r, A)\} \widetilde{\mp}\{(-r, A)\} \cong\{(-r, A)\} \widetilde{+}\{(r, A)\} \cong\{(0, A)\}, \\
& \{(r, A)\} \widetilde{\mp}\{(-r, \Phi)\} \cong\{(-r, \Phi)\} \widetilde{\mp}\{(r, A)\} \cong\{(0, A)\} .
\end{aligned}
$$

Similarly, $(\widetilde{\mathcal{R}}, \widetilde{P})$ is not a group.

(ii) $\left(\widetilde{\mathcal{R}}, \widetilde{+}_{\mathcal{R}}\right)$ is not a group, because for any soft real point $\{(r, A)\} \in \widetilde{\mathcal{R}}$, we have two identity soft real points $\{\{0, A\}\}$ and $\{(0, \mathcal{R})\}$. We have

$$
\begin{aligned}
& \{(r, A)\} \widetilde{\mp}_{\mathcal{R}}\{(0, A)\} \cong\{(0, A)\} \widetilde{\mp}_{\mathcal{R}}\{(r, A)\} \cong\{(r, A)\}, \\
& \{(r, A)\} \widetilde{\mp}_{\mathcal{R}}\{(0, R)\} \cong\{(0, R)\} \widetilde{+}_{\mathcal{R}}\{(r, A)\} \cong\{(r, A)\} .
\end{aligned}
$$

Similarly, $\left(\widetilde{\mathcal{R}}, \sim_{\mathcal{R}}\right)$ is not a group.

Proposition 12 If $\left(\widetilde{\mathcal{R}}, \widetilde{+}_{\Delta}\right)$ is a group, then, for every $\{(r, A)\},\{(s, B)\},\{(t, C)\} \in \widetilde{\mathcal{R}}$, we have the following properties:

(i) $\simeq(\simeq\{(r, A)\}) \cong\{(r, A)\}$,

(ii) $\simeq\left(\{(r, A)\} \widetilde{+}_{\Delta}\{(s, B)\}\right) \cong(\simeq\{(r, A)\}) \widetilde{\mp}_{\Delta}(\widetilde{\simeq}\{(s, B)\})$

$$
\cong(\simeq\{(s, B)\}) \widetilde{+}_{\Delta}(\widetilde{\simeq}\{(r, A)\}) .
$$

Proof (i) Since $(\widetilde{\simeq}\{(r, A)\}) \widetilde{+}_{\Delta}\{(r, A)\} \cong\{(0, \Phi)\} \cong\{(r, A)\} \widetilde{+}_{\Delta}(\widetilde{\simeq}\{(r, A)\})$ and $(\widetilde{\simeq}\{(r, A)\})$ is unique, $\{(r, A)\} \cong\{(0, \Phi)\} \widetilde{\mp}_{\Delta}(\widetilde{\simeq}(\widetilde{\sim}\{(r, A)\}))$. This implies that $\simeq(\widetilde{\sim}\{(r, A)\}) \cong\{(r, A)\}$.

(ii) $\simeq(\{(r, A)\} \widetilde{+} \Delta\{(s, B)\}) \cong \simeq\{(r+s, A \triangle B)\}$

$$
\begin{aligned}
& \cong\{(-(r+s), A \triangle B)\} \quad(\text { since } \simeq\{(r, A)\} \cong\{(-r, A)\}) \\
& \cong\{((-r)+(-s), A \triangle B)\} \cong\{(-r, A)\} \widetilde{f}_{\triangle}\{(-s, B)\} .
\end{aligned}
$$

Since $\left(\widetilde{\mathcal{R}}, \widetilde{+}_{\triangle}\right)$ is an abelian group,

$$
\begin{aligned}
\simeq\left(\{(r, A)\} \widetilde{+}_{\Delta}\{(s, B)\}\right) & \cong(\simeq\{(r, A)\}) \widetilde{\mp}_{\Delta}(\simeq\{(s, B)\}) \\
& \cong(\simeq\{(s, B)\}) \widetilde{\mp}_{\Delta}(\simeq\{(r, A)\}) .
\end{aligned}
$$

Proposition 13 (Cancellation law) If $\left(\widetilde{\mathcal{R}}, \widetilde{+}_{\Delta}\right)$ is an abelian group, then, for every $\{(r, A)\}$, $\{(s, B)\},\{(t, C)\} \in \widetilde{\mathcal{R}}$, we have:

(i) $\{(r, A)\} \widetilde{+}_{\Delta}\{(t, C)\} \cong\{(s, B)\} \widetilde{+}_{\Delta}\{(t, C)\} \Rightarrow\{(r, A)\} \cong\{(s, B)\}$, 
(ii) $\{(t, C)\} \widetilde{+}_{\Delta}\{(r, A)\} \cong\{(t, C)\} \widetilde{f}_{\Delta}\{(s, B)\} \Rightarrow\{(r, A)\} \cong\{(s, B)\}$.

Proof We note that

$$
\left(\{(r, A)\} \widetilde{+}_{\Delta}\{(t, C)\}\right) \widetilde{f}_{\Delta}(\{(-t, C)\}) \cong\left(\{(s, B)\} \widetilde{+}_{\Delta}\{(t, C)\}\right) \widetilde{+}_{\Delta}\{(-t, C)\} .
$$

This implies that

$$
\{(r, A)\} \widetilde{+}_{\Delta}\left(\{(t, C)\} \widetilde{+}_{\Delta}(\{(-t, C)\})\right) \cong\{(s, B)\} \widetilde{+}_{\Delta}\left(\{(t, C)\} \widetilde{+}_{\Delta}(\{(-t, C)\})\right) .
$$

This implies that $\{(r, A)\} \widetilde{\mp}_{\Delta}\{(0, \Phi)\} \cong\{(s, B)\} \widetilde{\mp}_{\Delta}\{(0, \Phi)\}$.

This implies that $\{(r, A)\} \cong\{(s, B)\}$.

(ii) Similar to (i).

Proposition 14 The group $\left(\widetilde{\mathcal{R}}, \widetilde{+}_{\Delta}\right)$ has two trivial subgroups, which are $\left(\{(0, \Phi)\}, \widetilde{+}_{\Delta}\right)$ and $\left(\widetilde{\mathcal{R}}, \widetilde{+}_{\Delta}\right)$.

Proposition 15 Let $\left(\widetilde{\mathcal{R}}, \widetilde{+}_{\Delta}\right)$ is an abelian group and $\widetilde{G}$ be set of some soft real points of $\widetilde{\mathcal{R}}$. Then we say that $\left(\widetilde{G}, \widetilde{+}_{\Delta}\right)$ is a proper subgroup of $\left(\widetilde{\mathcal{R}}, \widetilde{+}_{\Delta}\right)$ iff

(i) $\widetilde{G} \neq\{(0, \Phi)\}$,

(ii) $\left(\widetilde{G}, \widetilde{+}_{\Delta}\right)$ is a group.

Example 3 Let $\mathbb{Z}$ be the set of integer numbers and let $\widetilde{Z}=\{\{(z, A)\}, z \in Z$ and $A \subseteq Z\}$ be the set of soft integer points. Then we say that $\left(\widetilde{Z}, \widetilde{+}_{\Delta}\right)$ is a proper subgroup of $\left(\widetilde{\mathcal{R}}, \widetilde{+}_{\Delta}\right)$.

Proposition 16 Let $\widetilde{\mathcal{R}}$ be the set of soft real points and for all $\{(r, A)\},\left\{\left(r^{\prime}, A^{\prime}\right)\right\} \in \widetilde{\mathcal{R}}$, the binary operation on $\widetilde{\mathcal{R}}$ defined by

$$
\widetilde{\mathcal{R}} \times \widetilde{\mathcal{R}} \rightarrow \widetilde{\mathcal{R}}, \quad\left(\{(r, A)\},\left\{\left(r^{\prime}, A^{\prime}\right)\right\}\right) \rightarrow\{(r, A)\} \widetilde{+} \Delta\left\{\left(r^{\prime}, A^{\prime}\right)\right\} \cong\left\{\left(r+r^{\prime}, A \Delta A^{\prime}\right)\right\},
$$

where $\triangle$ is the symmetric difference of $A$ and $A^{\prime}$, and

$$
\widetilde{\mathcal{R}} \times \widetilde{\mathcal{R}} \rightarrow \widetilde{\mathcal{R}}, \quad\left(\{(r, A)\},\left\{\left(r^{\prime}, A^{\prime}\right)\right\}\right) \rightarrow\{(r, A)\} \widetilde{\sim}_{\mathcal{R}}\left\{\left(r^{\prime}, A^{\prime}\right)\right\} \cong\left\{\left(r \cdot r^{\prime}, A \cap A^{\prime}\right)\right\} .
$$

Then the triplet $\left(\widetilde{\mathcal{R}}, \widetilde{+}_{\Delta}, \widetilde{r}_{\mathcal{R}}\right)$ is a ring.

Proof $(1)\left(\widetilde{\mathcal{R}}, \widetilde{\mp}_{\Delta}\right)$ is an abelian group by Proposition 10.

(2) $\left(\widetilde{\mathcal{R}}, \widetilde{\bullet}_{\mathcal{R}}\right)$ is a semi group by Proposition 9 .

(3) We prove that the left and right distributive laws hold.

For all $\{(r, A)\},\{(s, B)\},\{(t, C)\} \in \widetilde{\mathcal{R}}$,

$$
\begin{aligned}
& \{(r, A)\} \widetilde{\sim}_{\mathcal{R}}\left(\{(s, B)\} \widetilde{\mp}_{\Delta}\{(t, C)\}\right) \\
& \quad \cong\{(r, A)\} \widetilde{\sim}_{\mathcal{R}}\{(s+t, B \Delta C)\} \\
& \quad \cong(\{(r \cdot(s+t), A \cap(B \Delta C))\}) \\
& \quad \cong(\{((r \cdot s)+(r \cdot t),(A \cap B) \Delta(A \cap C))\}) \\
& \quad \cong\left(\{(r, A)\} \widetilde{\sim}_{\mathcal{R}}\{(s, B)\}\right) \widetilde{\mp}_{\Delta}\left(\{(r, A)\} \widetilde{\sim}_{\mathcal{R}}\{(t, C)\}\right) .
\end{aligned}
$$


On the other hand,

$$
\begin{aligned}
& \left(\{(r, A)\} \widetilde{+}_{\Delta}\{(s, B)\}\right) \widetilde{\sim}_{\mathcal{R}}\{(t, C)\} \\
& \cong\{(r+s, A \triangle B)\} \widetilde{\sim}_{\mathcal{R}}\{(t, C)\} \\
& \cong(\{(r+s) \cdot t,(A \Delta B) \cap C\}) \\
& \cong(\{((r \cdot t)+(s \cdot t),(A \cap C) \Delta(B \cap C))\}) \\
& \cong\left(\{(r, A)\} \widetilde{\sim}_{\mathcal{R}}\{(t, C)\}\right) \widetilde{+}_{\Delta}\left(\{(s, B)\} \widetilde{\sim}_{\mathcal{R}}\{(t, C)\}\right) .
\end{aligned}
$$

Thus by (1), (2) and (3), we see that the triplet $\left(\widetilde{\mathcal{R}}, \widetilde{+}_{\Delta}, \widetilde{\cdot}_{\mathcal{R}}\right)$ is a ring.

Since $\left(\widetilde{\mathcal{R}}, \widetilde{\mathcal{R}}_{\mathcal{R}}\right)$ is not a group, the following proposition follows directly.

Proposition 17 The triplet $\left(\widetilde{\mathcal{R}}, \widetilde{+}_{\Delta}, \widetilde{r}_{\mathcal{R}}\right)$ is not a field.

Definition 25 Let $\widetilde{\mathcal{R}}$ be the set of all soft real points and $\widetilde{d}$ be a function from $\widetilde{\mathcal{R}} \times \widetilde{\mathcal{R}}$ to $\widetilde{\mathcal{R}}$. That is, $\widetilde{d}: \widetilde{\mathcal{R}} \times \widetilde{\mathcal{R}} \rightarrow \widetilde{\mathcal{R}}$. The function $\widetilde{d}$ is called a soft real point metric, if it satisfies the following conditions:

$$
\begin{aligned}
& \left(D_{1}\right) \widetilde{d}(\{(r, A)\},\{(s, B)\}) \widetilde{\simeq}\{(0, \Phi)\}, \\
& \left(D_{2}\right) \tilde{d}(\{(r, A)\},\{(s, B)\}) \cong\{(0, \Phi)\} \text { iff }\{(r, A)\} \cong\{(s, B)\}, \\
& \left(D_{3}\right) \tilde{d}(\{(r, A)\},\{(s, B)\}) \cong \widetilde{d}(\{(s, B)\},\{(r, A)\}), \\
& \left(D_{4}\right) \tilde{d}(\{(r, A)\},\{(t, C)\}) \widetilde{\leq} \widetilde{d}(\{(r, A)\},\{(s, B)\}) \widetilde{+} \widetilde{d}(\{(s, B)\},\{(t, C)\}) \text {, for all }\{(r, A)\},\{(s, B)\} \text {, } \\
& \{(t, C)\} \in \widetilde{\mathcal{R}} \text {. }
\end{aligned}
$$

Then the pair $(\widetilde{\mathcal{R}}, \widetilde{d})$ is called a soft real point metric space.

Example 4. Consider the function $\tilde{d}: \widetilde{\mathcal{R}} \times \widetilde{\mathcal{R}} \rightarrow \widetilde{\mathcal{R}}$ defined by

$$
\tilde{d}(\{(r, A)\},\{(s, B)\}) \cong|\{(r, A)\} \simeq\{(s, B)\}| .
$$

Then $(\widetilde{\mathcal{R}}, \widetilde{d})$ is a soft real point metric space.

For this we satisfy the properties of soft real point metric. Thus, for all $\{(r, A)\},\{(s, B)\}$, $\{(t, C)\} \in \widetilde{\mathcal{R}}$,

$$
\begin{aligned}
&\left(D_{1}\right) \quad \tilde{d}(\{(r, A)\},\{(s, B)\}) \\
& \cong|\{(r, A)\} \simeq\{(s, B)\}| \\
& \cong|\{(r-s, A \cup B)\}| \cong\{(|r-s|, A \cup B)\} \cong\{(0, \Phi)\} . \\
&\left(D_{2}\right) \quad \widetilde{d}(\{(r, A)\},\{(s, B)\}) \cong\{(0, \Phi)\} \\
& \Leftrightarrow|\{(r, A)\} \simeq\{(s, B)\}| \cong\{(0, \Phi)\} \\
& \Longleftrightarrow \quad|\{(r-s, A \cup B)\}| \cong\{(0, \Phi)\} \\
& \Longleftrightarrow \quad\{(|r-s|, A \cup B)\} \cong\{(0, \Phi)\} \quad \Longleftrightarrow \quad r-s=0 \text { and } A \cup B=\Phi \\
& \Longleftrightarrow \quad r=s \text { and } A=B=\Phi \quad \Longleftrightarrow \quad\{(r, A)\} \cong\{(s, B)\} . \\
&\left(D_{3}\right) \quad \widetilde{d}(\{(r, A)\},\{(s, B)\}) \\
& \quad \cong|\{(r, A)\} \simeq\{(s, B)\}|
\end{aligned}
$$




$$
\begin{aligned}
& \cong|\{(r-s, A \cup B)\}| \cong\{(|r-s|, A \cup B)\} \\
& \cong\{(|-(s-r)|, B \cup A)\} \cong\{(|(s-r)|, B \cup A)\} \\
& \cong \widetilde{d}(\{(s, B)\},\{(r, A)\}) . \\
&\left(D_{4}\right) \quad \widetilde{d}(\{(r, A)\},\{(s, B)\}) \widetilde{+} \widetilde{d}(\{(s, B)\},\{(t, C)\}) \\
& \cong|\{(r-s, A \cup B)\}| \widetilde{+}|\{(s-t, B \cup C)\}| \\
& \\
& \simeq|\{(r-s, A \cup B)\} \widetilde{+}\{(s-t, B \cup C)\}| \cong|\{(r-t, A \cup B \cup C)\}| \\
& \simeq|\{(r-t, A \cup C)\}| \cong \widetilde{d}(\{(r, A)\},\{(t, C)\}) .
\end{aligned}
$$

Definition 26 Let $(\widetilde{\mathcal{R}}, \widetilde{d})$ be a soft real point metric space. If there exists a positive soft real point $\{(t, C)\}$ such that $\widetilde{d}(\{(r, A)\},\{(s, B)\}) \widetilde{\leq}\{(t, C)\}$, for all soft real point $\{(r, A)\}$ and $\{(s, B)\}$, then $(\widetilde{\mathcal{R}}, \widetilde{d})$ is called a bounded soft metric space. Otherwise we consider it unbounded.

Definition 27 Let $\widetilde{\mathcal{R}}$ be the set of all soft real points and $\widetilde{d}$ be a function from $\widetilde{\mathcal{R}} \times \widetilde{\mathcal{R}}$ to $\widetilde{\mathcal{R}}$. That is $\widetilde{d}: \widetilde{\mathcal{R}} \times \widetilde{\mathcal{R}} \rightarrow \widetilde{\mathcal{R}}$. The function $\widetilde{d}$ is called a soft pseudo real point metric on the set of soft real points $\widetilde{\mathcal{R}}$, if it satisfies the following conditions:

$\left(D_{1}\right) \tilde{d}(\{(r, A)\},\{(s, B)\}) \simeq\{(0, \Phi)\}$ and $\widetilde{d}(\{(r, A)\},\{(r, A)\}) \cong\{(0, \Phi)\}$,

$\left(D_{2}\right) \tilde{d}(\{(r, A)\},\{(s, B)\}) \cong\{(0, \Phi)\}$, if $\{(r, A)\} \cong\{(s, B)\}$,

$\left(D_{3}\right) \tilde{d}(\{(r, A)\},\{(s, B)\}) \cong \widetilde{d}(\{(s, B)\},\{(r, A)\})$,

$\left(D_{4}\right) \tilde{d}(\{(r, A)\},\{(t, C)\}) \widetilde{\leq} \widetilde{d}(\{(r, A)\},\{(s, B)\}) \widetilde{+} \widetilde{d}(\{(s, B)\},\{(t, C)\})$, for all $\{(r, A)\},\{(s, B)\}$, $\{(t, C)\} \in \widetilde{\mathcal{R}}$.

Then the pair $(\widetilde{\mathcal{R}}, \widetilde{d})$ is called a soft pseudo real point metric space.

Example 5 Consider the function $\widetilde{d}: \widetilde{\mathcal{R}} \times \widetilde{\mathcal{R}} \rightarrow \widetilde{\mathcal{R}}$ defined by $\widetilde{d}(\{(r, A)\},\{(s, B)\}) \cong\{(0, \Phi)\}$, for all soft real point $\{(r, A)\}$ and $\{(s, B)\}$. Clearly $(\widetilde{\mathcal{R}}, \widetilde{d})$ is a soft pseudo real point metric space but not a soft real point metric space.

Definition 28 Let $(\widetilde{\mathcal{R}}, \widetilde{d})$ be a soft real point metric space. Then $\widetilde{\mathcal{A}}$ is called a soft real point subset of $\widetilde{\mathcal{R}}$, if $\{(r, A)\} \widetilde{\leq}\{(s, C)\}$, for each soft real point $\{(r, A)\}$ in $\widetilde{\mathcal{A}}$ and soft real point $\{(s, C)\}$ in $\widetilde{\mathcal{R}}$ with $r \leq c$ and $A \subseteq C$.

Definition 29 Let $(\widetilde{\mathcal{R}}, \widetilde{d})$ be a soft real point metric space and $\widetilde{\mathcal{A}}$ be a nonempty soft real point subset of $\widetilde{\mathcal{R}}$. Then the mapping $\widetilde{d_{\widetilde{\mathcal{A}}}}: \widetilde{\mathcal{A}} \times \widetilde{\mathcal{A}} \rightarrow \widetilde{\mathcal{R}}$ is defined by $\widetilde{d_{\widetilde{\mathcal{A}}}}(\{(r, A)\},\{(s, B)\}) \cong$ $\widetilde{d}(\{(r, A)\},\{(s, B)\})$, for all soft real point $\{(r, A)\}$ and $\{(s, B)\}$ in $\widetilde{\mathcal{A}}$. This metric $\widetilde{d_{\widetilde{A}}}$ is known as the relative soft real point metric induced on $\widetilde{d_{\widetilde{\mathcal{A}}}}$ by $\widetilde{d}$. The soft real point metric space $\left(\widetilde{\mathcal{A}}, \widetilde{d_{\widetilde{\mathcal{A}}}}\right)$ is called a soft real point metric subspace or simply a soft real point subspace of the soft real point metric space $(\widetilde{\mathcal{R}}, \widetilde{d})$.

Definition 30 Let $(\widetilde{\mathcal{R}}, \widetilde{d})$ be a soft real point metric space and $\widetilde{\mathcal{A}}$ be a nonempty soft real point subset of $\widetilde{\mathcal{R}}$. The diameter of $\widetilde{\mathcal{A}}$ is denoted by $\operatorname{diam}(\widetilde{\mathcal{A}})$ and is defined as

$$
\operatorname{diam}(\widetilde{\mathcal{A}}) \cong \sup \{\widetilde{d}(\{(r, A)\},\{(s, B)\}) \text {; where }\{(r, A)\} \text { and }\{(s, B)\} \text { are in } \widetilde{\mathcal{A}}\} .
$$

Clearly, $\operatorname{diam}(\widetilde{\mathcal{A}}) \widetilde{\geq}\{(0, \Phi)\}$. 
Definition 31 Let $(\widetilde{\mathcal{R}}, \widetilde{d})$ be a soft real point metric space, $r_{n} \in \mathcal{R}$ and $A \subseteq \mathcal{R}$. A sequence $\left\{\left(r_{n}, A\right)\right\}_{n}$ of soft real points in $\widetilde{\mathcal{R}}$ is said to be convergent in $(\widetilde{\mathcal{R}}, \widetilde{d})$, if there is a soft real point $\{(r, A)\}$ such that $\tilde{d}\left(\left\{\left(r_{n}, A\right)\right\}_{n},\{(r, A)\}\right) \rightarrow\{(0, \Phi)\}$ as $n \rightarrow \infty$. That is, for any arbitrary chosen $\widetilde{\epsilon} \cong\{(\epsilon, A)\} \widetilde{>}\{(0, \Phi)\}$, there exists $m \in \mathbb{N}$ such that $\widetilde{d}\left(\left\{\left(r_{n}, A\right)\right\}_{n},\{(r, A)\}\right) \widetilde{\epsilon} \widetilde{\text {, }}$ whenever $n \geq m$.

The proof of the following proposition follows immediately from the definition and thus is omitted.

Proposition 18 The limit of a soft real points sequence $\left\{\left(r_{n}, A\right)\right\}_{n}$ in a soft real point metric $\operatorname{space}(\widetilde{\mathcal{R}}, \widetilde{d})$, if it exists, is unique.

Definition 32 Let $(\widetilde{\mathcal{R}}, \widetilde{d})$ be a soft real point metric space, $r_{n}, r_{m} \in \mathcal{R}$ and $A \subseteq \mathcal{R}$. A sequence $\left\{\left(r_{n}, A\right)\right\}_{n}$ of soft real points in $\widetilde{\mathcal{R}}$ is said to be a Cauchy sequence in $(\widetilde{\mathcal{R}}, \widetilde{d})$, if, for each $\widetilde{\epsilon} \cong\{(\epsilon, A)\} \widetilde{>}\{(0, \Phi)\}$, there exists $p \in \mathbb{N}$ such that $\widetilde{d}\left(\left\{\left(r_{n}, A\right)\right\}_{n},\left\{\left(r_{m}, A\right)\right\}_{m}\right) \widetilde{\varepsilon}$, for all $n, m \geq p$. That is,

$$
\tilde{d}\left(\left\{\left(r_{n}, A\right)\right\}_{n},\left\{\left(r_{m}, A\right)\right\}_{m}\right) \rightarrow\{(0, \Phi)\} \quad \text { as } n, m \rightarrow \infty .
$$

Proposition 19 Every convergent sequence $\left\{\left(r_{n}, A\right)\right\}_{n}$ in a soft real point metric space $(\widetilde{\mathcal{R}}, \widetilde{d})$ is a Cauchy sequence in $(\widetilde{\mathcal{R}}, \widetilde{d})$.

Proof Let $(\widetilde{\mathcal{R}}, \widetilde{d})$ be a soft real point metric space, $r_{n}, r_{m}, r \in \mathcal{R}$ and $A \subseteq \mathcal{R}$. Suppose that the sequence $\left\{\left(r_{n}, A\right)\right\}_{n}$ of soft real points in $\widetilde{\mathcal{R}}$ is convergent. Let $\widetilde{\epsilon} \cong\{(\epsilon, A)\} \widetilde{>}$ $\{(0, \Phi)\}$ and $\widetilde{d}\left(\left\{\left(r_{n}, A\right)\right\}_{n},\{(r, A)\}\right) \rightarrow\{(0, \Phi)\}$ as $n \rightarrow \infty$. Then there exists $t \in \mathbb{N}$ such that $\widetilde{d}\left(\left\{\left(r_{n}, A\right)\right\}_{n},\{(r, A)\}\right) \widetilde{\epsilon}$, for all $n \geq t$.

Now for any $n, m \geq t$,

$$
\begin{aligned}
\widetilde{d}\left(\left\{\left(r_{n}, A\right)\right\}_{n},\left\{\left(r_{m}, A\right)\right\}_{m}\right) & \widetilde{\leq} \widetilde{d}\left(\left\{\left(r_{n}, A\right)\right\}_{n},\{(r, A)\}\right) \widetilde{+} \widetilde{d}\left(\left\{\left(r_{m}, A\right)\right\}_{m},\{(r, A)\}\right) \\
& \rightarrow\{(0, \Phi)\} \widetilde{+}\{(0, \Phi)\} \cong\{(0, \Phi)\}, \quad \text { as } n, m \rightarrow \infty .
\end{aligned}
$$

Thus

$$
\tilde{d}\left(\left\{\left(r_{n}, A\right)\right\}_{n},\left\{\left(r_{m}, A\right)\right\}_{m}\right) \rightarrow\{(0, \Phi)\} \quad \text { as } n, m \rightarrow \infty .
$$

This implies that $\left\{\left(r_{n}, A\right)\right\}_{n}$ is a Cauchy sequence in $(\widetilde{\mathcal{R}}, \widetilde{d})$. Hence the proof is completed.

The converse of the above proposition is not true in general.

Example 6 Suppose $\widetilde{\mathcal{R}_{1}} \cong\{(r, A): r \in(0,1], A \subseteq \mathcal{R}\}$ and a soft real point metric $\widetilde{d}$ from $\widetilde{\mathcal{R}_{1}} \times \widetilde{\mathcal{R}_{1}}$ to $\widetilde{\mathcal{R}}$ is defined by $\widetilde{d}(\{(r, A)\},\{(s, B)\}) \cong|\{(r, A)\} \simeq\{(s, B)\}|$.

Consider a soft real point sequence $\left\{\left(r_{n}, A\right)\right\}_{n} \cong\{(1 / n, A)\}_{n}$. Clearly $\left\{\left(r_{n}, A\right)\right\}_{n} \cong\{(1 / n, A)\}_{n}$ is a Cauchy sequence in $\widetilde{\mathcal{R}_{1}}$. But it is not convergent in $\widetilde{\mathcal{R}_{1}}$, since $\left\{\left(r_{n}, A\right)\right\}_{n} \cong\{(1 / n, A)\}_{n} \rightarrow$ $\{(0, A)\}$. But $\{(0, A)\} \widetilde{\in} \widetilde{\mathcal{R}_{1}}$.

Definition 33 Let $(\widetilde{\mathcal{R}}, \widetilde{d})$ be a soft real point metric space. Then $(\widetilde{\mathcal{R}}, \widetilde{d})$ is called soft real point complete, if every Cauchy sequence in $\widetilde{\mathcal{R}}$ converges to some soft real point of $\widetilde{\mathcal{R}}$. 
Example 7 In Example 6, $\left(\widetilde{\mathcal{R}_{1}}, \widetilde{d}\right)$ is not soft real point complete.

Definition 34 Let $(\widetilde{\mathcal{R}}, \widetilde{d})$ be a soft real point metric space and $\{(r, A)\}$ be a non-negative soft real point. For any $\{(s, B)\}$ soft real point in $\widetilde{\mathcal{R}}$, we define the soft real point open ball with centre $\{(s, B)\}$ and radius $\{(r, A)\}$ denoted by $B(\{(s, B)\},\{(r, A)\})$ and it is defined as the collection of soft real points $\{(t, C)\}$ of $\widetilde{\mathcal{R}}$ which satisfy $\widetilde{d}(\{(t, C)\},\{(s, B)\}) \widetilde{<}(r, A)$. Thus

$$
B(\{(s, B)\},\{(r, A)\}) \cong\{\{(t, C)\} \widetilde{\in} \widetilde{\mathcal{R}} ; \widetilde{d}(\{(t, C)\},\{(s, B)\}) \widetilde{<}(r, A)\} \widetilde{\subseteq} \widetilde{\mathcal{R}} .
$$

Definition 35 Let $(\widetilde{\mathcal{R}}, \widetilde{d})$ be a soft real point metric space and $\{(r, A)\}$ be a non-negative soft real point. For any $\{(s, B)\}$ soft real point in $\widetilde{\mathcal{R}}$, we define the soft real point closed ball with centre $\{(s, B)\}$ and radius $\{(r, A)\}$ denoted by $B[\{(s, B)\},\{(r, A)\}]$ and it is defined as the collection of soft real points $\{(t, C)\}$ of $\widetilde{\mathcal{R}}$ which satisfy $\widetilde{d}(\{(t, C)\},\{(s, B)\}) \widetilde{\leq}(r, A)$. Thus

$$
B[\{(s, B)\},\{(r, A)\}] \cong\{\{(t, C)\} \widetilde{\in} \widetilde{\mathcal{R}} ; \widetilde{d}(\{(t, C)\},\{(s, B)\}) \widetilde{\leq}(r, A)\} \widetilde{\subseteq} \widetilde{\mathcal{R}} .
$$

Definition 36 Let $(\widetilde{\mathcal{R}}, \widetilde{d})$ be a soft real point metric space and $\{(s, A)\}$ be a soft real point. A collection $N(\{(s, A)\})$ of soft real points containing the soft real point $\{(s, A)\}$ is said to be soft real point neighbourhood of the soft real point $\{(s, A)\}$, if there exists a positive soft real point $\{(r, A)\}$ such that $\{(s, A)\} \widetilde{\in} B[\{(s, A)\},\{(r, A)\}] \widetilde{\subseteq} N(\{(s, A)\})$. We will denote the soft real point neighbourhood of the soft real point $\{(s, A)\}$ as $N(\{(s, A)\})$.

The proof of the following theorem directly follows from the definition and is therefore omitted.

Theorem 1 Let $(\widetilde{\mathcal{R}}, \widetilde{d})$ be a soft real point metric space and $\{(s, A)\}$ be a soft real point. Suppose $N_{1}(\{(s, A)\})$ and $N_{2}(\{(s, A)\})$ are soft real point neighbourhoods of the soft real point $\{(s, A)\}$ in $(\widetilde{\mathcal{R}}, \widetilde{d})$. Then $N_{1}(\{(s, A)\}) \widetilde{\cap} N_{2}(\{(s, A)\})$ is also a soft real point neighbourhood of the soft real point $\{(s, A)\}$ in $(\widetilde{\mathcal{R}}, \widetilde{d})$.

Theorem 2 Every soft real point open ball is a soft real point neighbourhood of each of its soft real points.

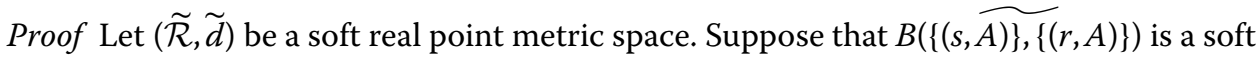
real point open ball with centre $\{(s, A)\}$ and radius $\{(r, A)\}$. Using the definition of a soft real point neighbourhood, it follows that $B(\{(s, \widehat{A})\},\{(r, A)\})$ is a soft real point neighbourhood of $\{(s, A)\}$ in $(\widetilde{\mathcal{R}}, \widetilde{d})$. Let us consider a soft real point $\{(t, A)\}$ in $B(\{(s, \widetilde{A})\},\{(r, A)\})$ different from $\{(s, A)\}$. Then we must have $\{(0, \Phi)\} \widetilde{\neq} \widetilde{d}(\{(t, A)\},\{(s, A)\}) \widetilde{\leq}\{(r, A)\}$. Choose $\left\{\left(r_{1}, A\right)\right\}$ with $\{(0, \Phi)\} \widetilde{\leq}\left\{\left(r_{1}, A\right)\right\} \widetilde{\leq}\{(r, A)\} \widetilde{\simeq}(\{(t, A)\},\{(s, A)\})$. It follows that $\left\{\left(r_{1}, A\right)\right\}$ is a positive

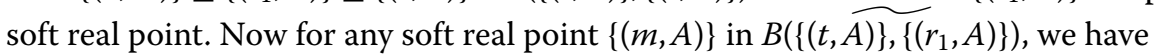

$$
\widetilde{d}(\{(t, A)\},\{(m, A)\}) \widetilde{\leq}\left\{\left(r_{1}, A\right)\right\} .
$$

Now

$$
\begin{aligned}
& \tilde{d}(\{(s, A)\},\{(m, A)\}) \\
& \quad \widetilde{\leq} \widetilde{d}(\{(s, A)\},\{(t, A)\}) \widetilde{+} \widetilde{d}(\{(t, A)\},\{(m, A)\}) \\
& \quad \widetilde{\leq} \widetilde{d}(\{(s, A)\},\{(t, A)\}) \widetilde{+}\left\{\left(r_{1}, A\right)\right\} \widetilde{\leq}\{(r, A)\} .
\end{aligned}
$$


Therefore,

$$
\{(m, A)\} \widetilde{\in} B(\{(s, \widetilde{A)}\},\{(r, A)\}) .
$$

This implies that

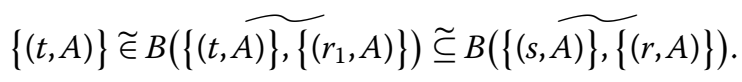

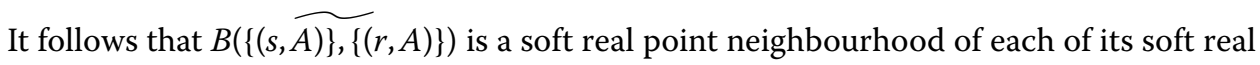
points. This completes the proof.

Definition 37 Let $\widetilde{\mathcal{R}}$ and $\widetilde{\mathcal{R}_{1}}$ be two sets of soft real points and $T: \widetilde{\mathcal{R}} \widetilde{\longrightarrow} \widetilde{\mathcal{R}_{1}}$ be a soft real point mapping. The image of $\widetilde{\mathcal{R}_{2}} \widetilde{\subseteq} \widetilde{\mathcal{R}}$ under the soft real point mapping $T$ is the soft real point set, denoted by $T\left(\widetilde{\mathcal{R}_{2}}\right)$, defined as follows:

$$
T\left(\widetilde{\mathcal{R}_{2}}\right) \cong \widetilde{\bigcup}\left\{T(\{(r, A)\}):\{(r, A)\} \widetilde{\in} \widetilde{\mathcal{R}_{2}}\right\} .
$$

Definition 38 Let $\widetilde{\mathcal{R}}$ and $\widetilde{\mathcal{R}_{1}}$ are two sets of soft real points and $T: \widetilde{\mathcal{R}} \widetilde{\longrightarrow} \widetilde{\mathcal{R}_{1}}$ be a soft real point mapping. The inverse image of $\widetilde{\mathcal{R}_{3}} \widetilde{\subseteq} \widetilde{\mathcal{R}_{1}}$ under the soft real point mapping $T$ is the soft real point set, denoted by $T^{-1}\left(\widetilde{\mathcal{R}_{3}}\right)$, defined as follows:

$$
T^{-1}\left(\widetilde{\mathcal{R}_{3}}\right) \cong \widetilde{\bigcup}\left\{T^{-1}(\{(s, A)\}):\{(s, A)\} \widetilde{\in \mathcal{R}_{3}}\right\} .
$$

Definition 39 Let $\widetilde{\mathcal{R}}$ be a set of soft real points and $T: \widetilde{\mathcal{R}} \widetilde{\longrightarrow} \widetilde{\mathcal{R}}$ be a soft real point mapping. A soft real point $\{(s, A)\} \widetilde{\in} \widetilde{\mathcal{R}}$ is called a soft real fixed point of $T$, if $T(\{(s, A)\})=$ $\{(s, A)\}$.

Definition 40 Let $(\widetilde{\mathcal{R}}, \widetilde{d})$ and $\left(\widetilde{\mathcal{R}_{1}}, \widetilde{d}_{1}\right)$ be two soft real point metric spaces. A soft real point mapping $T: \widetilde{\mathcal{R}} \underset{\longrightarrow}{\widetilde{\mathcal{R}_{1}}}$ is said to be a soft continuous at a soft real point $\{(s, A)\}$ in $(\widetilde{\mathcal{R}}, \widetilde{d})$, if, for every soft real point $\widetilde{\epsilon} \cong\{(\epsilon, A)\} \widetilde{>} \widetilde{0} \cong\{(0, \Phi)\}$, there exists a soft real point $\widetilde{\delta} \cong\{(\delta, A)\} \widetilde{>} \widetilde{0} \cong\{(0, \Phi)\}$ such that

$$
\widetilde{d}(\{(s, A)\},\{(m, A)\}) \widetilde{<} \widetilde{\delta} \text { implies } \widetilde{d}_{1}(T(\{(s, A)\}), T(\{(m, A)\})) \widetilde{\epsilon} .
$$

Note that if $T$ is soft continuous at every soft real point of $\widetilde{\mathcal{R}}$, then we say that $T$ is soft continuous on $\widetilde{\mathcal{R}}$.

Definition 41 Let $(\widetilde{\mathcal{R}}, \widetilde{d})$ be a soft real point metric space and $T: \widetilde{\mathcal{R}} \widetilde{\longrightarrow} \widetilde{\mathcal{R}}$ be a soft continuous mapping. Then $T$ is said to be a soft contraction if $\widetilde{d}(T(\{(s, A)\}), T(\{(m, A)\})) \widetilde{\leq}$ $\widetilde{c} \tilde{d}(\{(s, A)\},\{(m, A)\})$, for all $\{(s, A)\},\{(m, A)\} \widetilde{\in} \widetilde{\mathcal{R}}$ and $\{(0, \Phi)\} \widetilde{\leq} \widetilde{c} \cong\{(c, A)\} \widetilde{\leq}\{(1, A)\}$. The constant $\tilde{c}$ will be called a soft real point contraction constant. Note that the soft real point contraction on a soft real point metric space is a soft continuous mapping.

Now we construct our soft real point contraction fixed point theorem.

Theorem 3 Let $(\widetilde{\mathcal{R}}, \widetilde{d})$ be a soft real point complete metric space, where $\widetilde{d}: \widetilde{\mathcal{R}} \times \widetilde{\mathcal{R}} \rightarrow \widetilde{\mathcal{R}^{+}}$ and $\widetilde{\mathcal{R}^{+}} \cong\left\{\{(r, A)\} ; r \in \mathcal{R}^{+}, A \subseteq \mathcal{R}\right\}$. Suppose $T$ is a soft real point contraction with soft real point contraction constant $\widetilde{c} \cong\{(c, A)\}$. Then $T$ has a unique soft real fixed point $\{(m, A)\}$. 
Proof We choose a soft real point $\{(s, A)\}$. Take $\left\{\left(s_{0}, A\right)\right\} \cong\{(s, A)\}$ and note that

$$
\begin{aligned}
& \tilde{d}\left(\left\{\left(s_{n+1}, A\right)\right\}_{n},\left\{\left(s_{n}, A\right)\right\}_{n}\right) \\
& \quad \simeq \widetilde{d}\left(T\left(\left\{\left(s_{n}, A\right)\right\}_{n}\right), T\left(\left\{\left(s_{n-1}, A\right)\right\}_{n}\right)\right) \\
& \quad \widetilde{\leq} \widetilde{c}\left(\left\{\left(s_{n}, A\right)\right\}_{n},\left\{\left(s_{n-1}, A\right)\right\}_{n}\right) \\
& \quad \widetilde{\leq} \widetilde{c}^{2} \widetilde{d}\left(\left\{\left(s_{n-1}, A\right)\right\}_{n},\left\{\left(s_{n-2}, A\right)\right\}_{n}\right) \widetilde{\leq} \ldots \widetilde{\leq} \widetilde{c}^{n} \widetilde{d}\left(\left\{\left(s_{1}, A\right)\right\},\left\{\left(s_{0}, A\right)\right\}\right) .
\end{aligned}
$$

For $n>m$, we have

$$
\begin{aligned}
\widetilde{d} & \left(\left\{\left(s_{n}, A\right)\right\}_{n},\left\{\left(s_{m}, A\right)\right\}_{m}\right) \\
& \widetilde{\leq} \widetilde{d}\left(\left\{\left(s_{n}, A\right)\right\}_{n},\left\{\left(s_{n-1}, A\right)\right\}_{n}\right) \widetilde{+} \widetilde{d}\left(\left\{\left(s_{n-1}, A\right)\right\}_{n},\left\{\left(s_{n-2}, A\right)\right\}_{n}\right) \\
& \widetilde{+} \ldots \widetilde{+} \widetilde{d}\left(\left\{\left(s_{m+1}, A\right)\right\}_{m},\left\{\left(s_{m}, A\right)\right\}_{m}\right) \\
& \widetilde{\leq}\left(\widetilde{c}^{n-1} \widetilde{+} \widetilde{c}^{n-2} \widetilde{+} \ldots \widetilde{+} \widetilde{c}^{m}\right) \widetilde{d}\left(\left\{\left(s_{1}, A\right)\right\},\left\{\left(s_{0}, A\right)\right\}\right) \rightarrow\{(0, \Phi)\} \text { as } n \rightarrow \infty .
\end{aligned}
$$

Thus $\left\{\left(s_{m}, A\right)\right\}_{m}$ is a Cauchy sequence. As regards the completeness of $(\widetilde{\mathcal{R}}, \widetilde{d})$, there is a soft real point $\{(r, A)\}$ in $(\tilde{\mathcal{R}}, \tilde{d})$ such that $\tilde{d}\left(\left\{\left(s_{n}, A\right)\right\}_{n},\{(r, A)\}\right) \rightarrow\{(0, \Phi)\}$ as $n \rightarrow \infty$. We have

$$
\begin{aligned}
& \tilde{d}(\{(r, A)\}, T(\{(r, A)\})) \\
& \quad \widetilde{\leq} \widetilde{d}\left(\left\{\left(s_{n}, A\right)\right\}_{n},\{(r, A)\}\right) \widetilde{+} \widetilde{d}\left(\left\{\left(s_{n}, A\right)\right\}_{n}, T(\{(r, A)\})\right) \\
& \quad \widetilde{\leq} \widetilde{d}\left(\left\{\left(s_{n}, A\right)\right\}_{n},\{(r, A)\}\right) \widetilde{+} \widetilde{c} \widetilde{d}\left(\left\{\left(s_{n-1}, A\right)\right\}_{n},\{(r, A)\}\right) .
\end{aligned}
$$

That $\tilde{d}\left(\left\{\left(s_{n}, A\right)\right\}_{n},\{(r, A)\}\right) \rightarrow\{(0, \Phi)\}$ as $n \rightarrow \infty$ implies that

$$
\tilde{d}\left(\left\{\left(s_{n-1}, A\right)\right\}_{n},\{(r, A)\}\right) \rightarrow\{(0, \Phi)\} \quad \text { as } n \rightarrow \infty .
$$

Therefore,

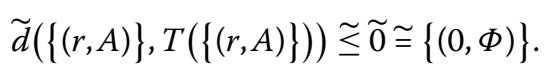

It follows that $T(\{(r, A)\}) \cong\{(r, A)\}$. Hence $\{(r, A)\}$ is the soft real point fixed point of $T$.

Now we prove uniqueness of $\{(r, A)\}$. For this, if we take another soft real fixed point $\{(u, A)\}$ of $T$, then

$$
\widetilde{d}(\{(r, A)\},\{(t, A)\}) \cong \widetilde{d}(T(\{(r, A)\}), T(\{(t, A)\})) \widetilde{\simeq} \widetilde{c} \widetilde{d}(\{(r, A)\},\{(t, A)\}) .
$$

Since $\widetilde{c} \widetilde{\leq}\{(1, A)\}$, we have $\{(r, A)\} \cong\{(t, A)\}$. Hence the fixed point of $T$ is unique. This completes the proof.

\section{Conclusions}

The idea of soft single points proceeding towards soft real points arose during the study of soft set theory; especially, soft topology and soft version of the usual topology on real numbers. We introduced and discussed soft single points, which proceed towards soft real points by using real numbers and subsets of a set of real numbers. We also defined the 
basic operations on soft real points and explored the algebraic properties. It is observed that the set of all soft real points forms a ring. Moreover, the soft real point metric is defined by using soft real points and we investigated some of its properties. The soft real point contraction fixed point theorem using a soft real point metric space is established. It is mentionable that these concepts may be helpful for researchers to navigate the ideas put forth in a soft metric extension of several important fixed point theorems for metric spaces deduced from comparable existing results.

Acknowledgements

Authors are thankful to the anonymous referees and the editor for their valuable comments towards improvement of this paper and rewriting it in present form.

Funding

We have no funding for this project of research.

\section{Abbreviations}

Not applicable.

\section{Availability of data and materials}

Please contact the authors for data requests.

\section{Competing interests}

The authors declare that they have no competing interests.

\section{Authors' contributions}

All authors contributed equally. All authors read and approved the final manuscript.

\section{Author details}

'Department of Mathematics, College of Science, Qassim University, Buraydah, Saudi Arabia. ${ }^{2}$ Department of Mathematics, Bozok University, Yozgat, Turkey.

\section{Publisher's Note}

Springer Nature remains neutral with regard to jurisdictional claims in published maps and institutional affiliations.

Received: 28 May 2018 Accepted: 5 May 2019 Published online: 20 May 2019

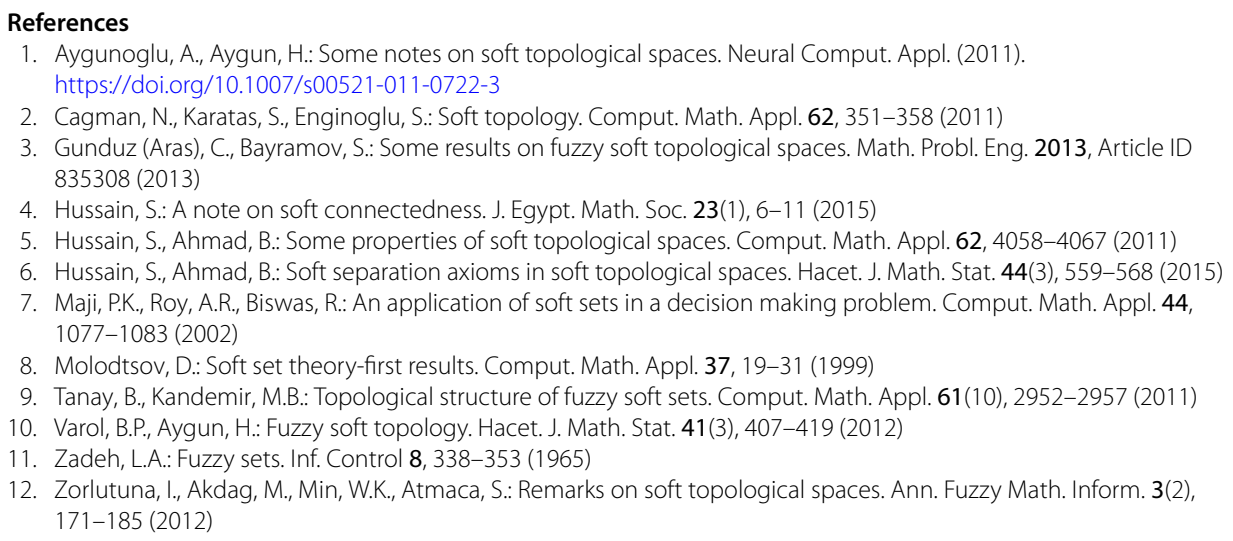

